\title{
Couf $-710.5239--2$
}

LBL-31382

\section{Lawrence Berkeley Laboratory UNIVERSITY OF CALIFORNIA}

\section{Physics Division}

Contribution to the Festschrift on the occasion of M. Veltman's 60th birthday, University of Michigan, Ann Arbor, MI, May 15-17, 1991, and to be published in the Proceedings

\section{Example of a Quantum Field Theory Based on a Nonlinear Lie Algebra}

K. Schoutens, A. Sevrin, and P. van Nieuwenhuizen

November 1991

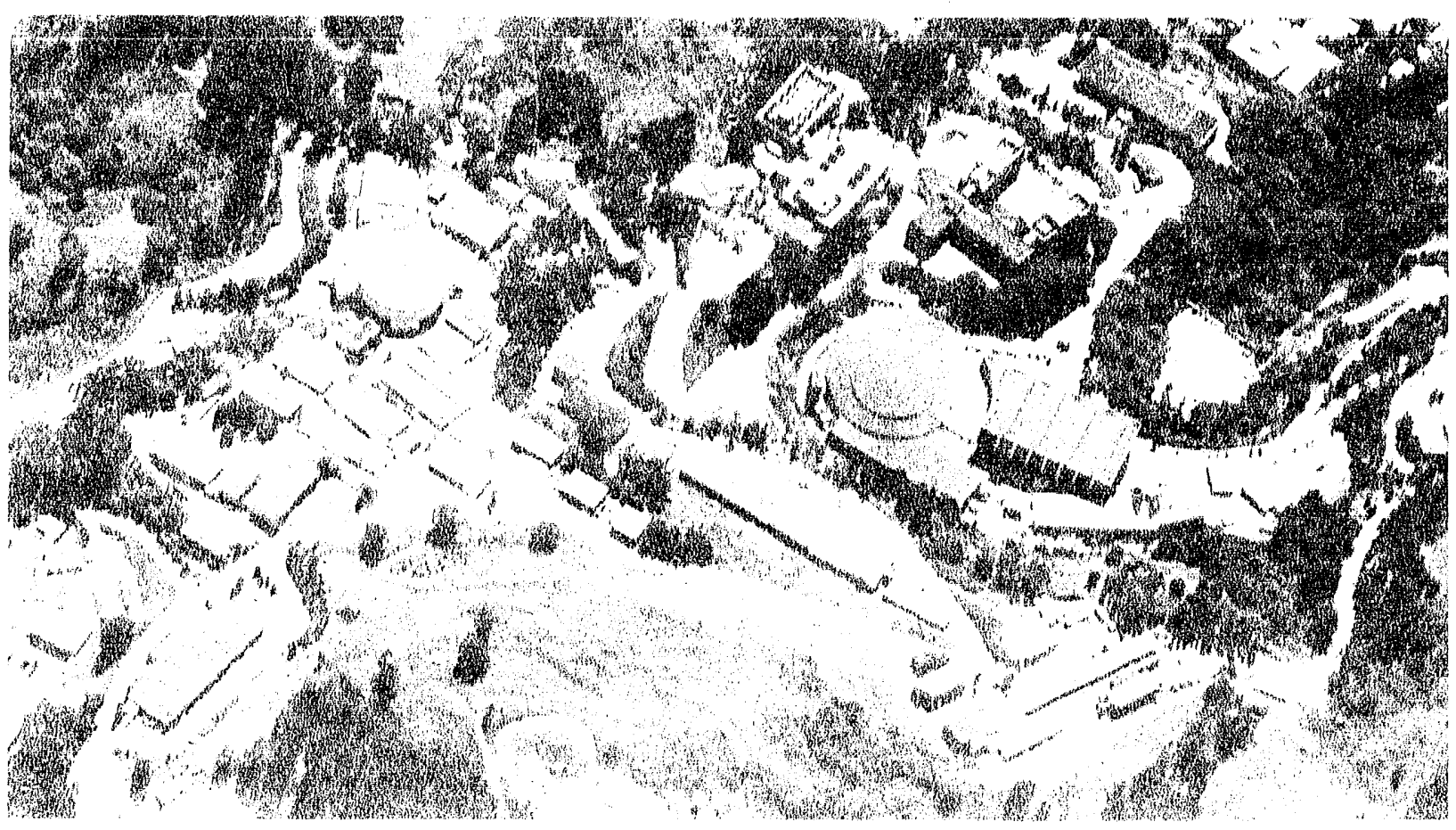

Prepared for the U.S. Department of Energy under Contract Number DE-AC03-76SF00098 


\section{DISCLAIMER}

This document was prepared as an account of work sponsored by the United States Government. Neither the United States Government nor any agency thereof, nor 'The Regents of the University of California, nor any of their employees, makes any warranty, express or inplied, or assumes any legal liability or responsibility for the accuracy, completeness, or usefulness of any information, apparatus, product, or process disclosed, or represents that its use would not infringe privately owned rights. Reference herein to any specific commercial product, process, or service by its trade name, trademark, manufacturer, or otherwise, does not necessarily constitute or imply its endorsement, recommendation, or favoring by the United States Govermment or any agency thereof, or The Regents of the University of California. The views and opinions of authors expressed herein do not necessarily state or reflect those of the United States Covernment or any agency thereof or The Regents of the University of California and shall not be used for advertising or product endorsement purposes.

Lawrence Berkeley Laboratory is an equal opportunity employer. 


\title{
Example of a quantum field theory based on a nonlinear Lie algebra ${ }^{1}$
}

\author{
K. Schoutens ${ }^{2}$ \\ Institute for Theoretical Physics \\ State University of New York at Stony Brook \\ Stony Brook, NY 11794-3840 \\ A. Sevrin ${ }^{3}$ \\ Department of Physics, University of California \\ and \\ Berkeley, California 94720 \\ and \\ P. van Nieuwenhuizen ${ }^{2,4}$ \\ Theory Division, CERN \\ CH-1211 Geneva 23 \\ Switzerland
}

Theoretical Physics Group, Lawrence Berkeley Laboratory

\footnotetext{
${ }^{1}$ Contribution to the Festschrift on the occasion of M. Veltman's 60th birthday, May 1991.

${ }^{2}$ Work supported in part by grant NSF-91-08054

${ }^{3}$ This work was supported in part by the Director, Office of Energy Research, Office of High Energy and Nuclear Physics, Division of High Energy Physics of the U.S. Department of Energy under Contract DE-AC03-76SF00098 and in part by the National Science Foundation under grant PHY90-21139.

${ }^{4}$ On leave from ITP, SUNY at Stony Brook
} 


\section{Introduction.}

In this contribution to Tini Veltman's Festschrift we shall give a paedagogical account of our work [1]-[6] on a new class of gauge theories called $W$ gravities. They contain higher spin gauge fields, but the usual no-go theorems for interacting field theories with spins exceeding two do not apply since these theories are in two dimensions. It is, of course, well known that ghost-free interacting massless spin 2 fields ('the metric') are gauge fields, and correspond to the geometrical notion of general coordinate transformations in general relativity, but it is yet unknown what extension of these ideas is introduced by the presence of massless higher spin gauge fields. A parallel with supergravity may be drawn: there the presence of massless spin $3 / 2$ fields (gravitinos) corresponds to local fermi-bose symmetries of which these gravitinos are the gauge fields. Their geometrical meaning becomes only clear if one introduces superspace (with bosonic and fermionic coordinates): they correspond to local transformations of the fermionic coordinates. For $W$ gravity one might speculate on a kind of $W$-superspace with extra bosonic coordinates. $^{5}$

A reason for being interestet in $W$ gravities is that they are, like ordinary $d=2$ gravity, 'integrable'. The integrability of these theories makes it possible to find exact answers, to all order in the number of loops, for various quantities in the theory such as the effective action. With such exact, all-order-in-perturbation-theory results one might start the study of nonperturbative properties. There one expects a connection with the so-called matrix models but at this moment no precise relations are known.

In this contribution we focus on (classical and quantum) $W_{3}$ gravity, which is the simplest version of $W$ gravity and contains, besides the spin 2 gravity fields, gauge fields of spin 3. The main part of our review will be to discuss how the effective action

\footnotetext{
${ }^{5}$ Incidentally, whether he likes it or not, Tini Veltman contributed indirectly to the discovery of supergravity. In 1974 he and Gerard 't Hooft had computed the 1-loop divergences of pure gravity (which was finite), and gravity coupled to scalars, (which was not finite) using their new covariant quantization methods. Their work was extended by various people, mostly Deser and one of us. We calculated the one-loop divergences of all kinds of different matter systems. None was finite. In this connection, Tini suggested in the fall of 1975 to consider the coupling of spin $3 / 2$ fields to gravity. This theory became supergravity. It was first constructed as the classical gauge theory of supersymmetry, but later it was indeed found that at the quantum level it was 1-loop finite. Now that recent results ([7]) confirm earlier work ([8]) that gravity is not 2-loop finite, many people believe that supergravity is not 3-loop finite, and that one should go to string theory for a consistent quantum theory of gravity. However, a proof that supergravity is not 3-loop finite is lacking.
} 
of this theory can be obtained from 'the $c \rightarrow \infty$ limit of the induced action' (which we explicitly found) by a renormalization of the spin 2 and spin 3 gauge fields and the $S l(3, \mathbf{R})$ level $k$ (with $Z$ factors which are infinite series in $c^{-1}$ ).

Pure gravity in $d=2$ is based on a linear algebra, the Virasoro algebra, which is given by

$$
\left[L_{m}, L_{n}\right]=(m-n) L_{m+n}, \quad m, n \in \mathbf{Z} .
$$

In contrast, $W_{3}$ gravity is based on a nonlinear algebra. The precise form will be given in the next section, but let us mention that its classical (Poisson bracket) version is of the form

$$
\left[T_{A}, T_{B}\right]=f_{A B}{ }^{C} T_{C}+V_{A B}^{C D} T_{C} T_{D}
$$

The work on $W_{3}$ gravity can therefore be considered as work on a prototype model for nonlinear gauge theories. Further models based on nonlinear algebras might be searched for and studied.

In [9], we have extended our work on non-linear gauge theories to four dimensions, where we have found that the kind of gauge theories we consider necessarily contain one real scalar field for each gauge field, and we have studied whether these scalars could act as Higgs scalars. [Scalar fields arise in general in gauge field theories based on nonlinear algebras. For example, consider the algebra given in (1.2). It yields, upon 'gauging', gauge fields $h_{\mu}{ }^{A}$ corresponding to the generators $T_{A}$. However, the presence of the $T T$ term in the abstract algebra leads, in addition, to scalar fields $t_{A}$ in the coadjoint representation of the gauge group. As such these scalar fields are an integral part of the gauge multiplet. In $W_{3}$ gravity, the scalar fields are $t_{++}\left(x^{+}, x^{-}\right)$and $w_{+++}\left(x^{+}, x^{-}\right)$ and look like currents. We have found [5] that they play the role of auxiliary fields needed to close the gauge algebra.] We intend to follow up on that work in the future, and shall not discuss it here as we already need a lot of clemency from the side of the 'jubilaris', who, of course, has probed the Higgs problem deeper than anybody else over the years. ${ }^{6}$

\footnotetext{
${ }^{6}$ In fact, as mentioned by Cabibbo, it is remarkable that one physicist (i) made such an important theoretical discovery as quantization of nonabelian gauge theories, (ii) then applied this theoretical framework to realistic models, doing much explicit numerical work useful for experiments, and (iii) developed his own software programs ('Schoonschip') and P.C.'s. With such a set of accomplishments
} 
Let us now stop enumerating the reasons why it is interesting to study $W$ gravities, as the relevance of a subject is inversely proportional to the number of such reasons. Instead, we start our discussion of $W_{3}$ gravity. We shall first, in sections 2 and 3 , discuss the $W_{3}$ algebra and various classical theories of $W_{3}$ gravity. In subsequent sections we shall then develop in some detail the quantum theory of $W_{3}$ gravity in the chiral gauge.

\section{The $W_{3}$ algebra, subalgebras and classical limits.}

The quantum $W_{3}$ algebra [10] contains the Virasoro algebra and further spin 3 generators $W_{m}$,

$$
\begin{aligned}
{\left[L_{m}, L_{n}\right] } & =\frac{c}{12} m\left(m^{2}-1\right) \delta_{m+n, 0}+(m-n) L_{m+n} \\
{\left[L_{m}, W_{n}\right] } & =(2 m-n) W_{m+n}
\end{aligned}
$$

which satisfy a quadratically nonlinear quantum algebra

$$
\begin{aligned}
{\left[W_{m}, W_{n}\right]=} & \frac{c}{360} m\left(m^{2}-1\right)\left(m^{2}-4\right) \delta_{m+n, 0} \\
& +(m-n)\left\{\frac{1}{15}(m+n+3)(m+n+2)-\frac{1}{6}(m+2)(n+2)\right\} L_{m+n} \\
& +\beta(m-n) \Lambda_{m+n} .
\end{aligned}
$$

In order that the Jacobi identities be satisfied, the central charge $c$ in the $[W, W]$ commutators must be the same as in the Virasoro algebra, and further

$$
\beta=\frac{16}{22+5 c}
$$

The objects $\Lambda_{m}$ are nonlinear in $L_{m}$

$$
\Lambda_{m}=\sum_{n}: L_{m-n} L_{n}:-\frac{3}{10}(m+3)(m+2) L_{m}
$$

he is entitled to his criticism of modern speculative theoretical developments. However, in the absence of obvious, fundamental and solvable problems in the standard model, it seems to us that quantum gravity stands out as the challenge of the next century. 
where the normal ordered product ${ }^{7}$ is defined as

$$
\begin{array}{ll}
: L_{p} L_{q}:=L_{p} L_{q} & \text { if } \quad p \leq-2 \\
: L_{p} L_{q}:=L_{q} L_{p} & \text { if } \quad p>-2 .
\end{array}
$$

They satisfy

$$
\left[L_{m}, \beta \Lambda_{n}\right]=(3 m-n) \beta \Lambda_{m+n}+\frac{8}{15}\left(m^{3}-m\right) L_{m+n}
$$

and, were it not for the last term, they would be primary fields of dimension 4 .

In terms of operator fields

$$
\begin{aligned}
& L(z)=\sum L_{m} z^{-m-2}, \quad W(z)=\sum W_{m} z^{-m-3} \\
& \Lambda(z)=(T T)(z)-\frac{3}{10} T^{\prime \prime}(z)=\sum \Lambda_{m} z^{-m-4}
\end{aligned}
$$

one can write down operator product expansions (OPE's), which are equivalent to (2.1), $(2.2)$

$$
\begin{aligned}
L(z) L(w)= & \frac{c / 2}{(z-w)^{4}}+\frac{2 T(w)}{(z-w)^{2}}+\frac{T^{\prime}(w)}{z-w}+\ldots \\
L(z) W(w)= & \frac{3 W(w)}{(z-w)^{2}}+\frac{W^{\prime}(w)}{z-w}+\ldots \\
W(z) W(w)= & \frac{c / 3}{(z-w)^{6}}+\frac{2 T(w)}{(z-w)^{4}}+\frac{T^{\prime}(w)}{(z-w)^{3}} \\
& +\frac{1}{(z-w)^{2}}\left[2 \beta \Lambda(w)+\frac{3}{10} T^{\prime \prime}(w)\right] \\
& +\frac{1}{z-w}\left[\beta \Lambda^{\prime}(w)+\frac{1}{15} T^{\prime \prime \prime}(w)\right]+\ldots
\end{aligned}
$$

We now observe the following.

(i) The subset of generators $w=\left\{L_{ \pm 1}, L_{0}, W_{ \pm 2}, W_{ \pm 1}, W_{0}\right\}$ does not form a subalgebra if $\beta \neq 0$.

${ }^{7}$ Different authors use different conventions for normal ordering, and consequently obtain slightly different forms of the algebra. Our normal ordering conventions follow from (6.10) and the mode expansions as in (2.7). 
(ii) The half-infinite sets $\left\{L_{m}, m \geq-1\right\}$ and $\left\{W_{n}, n \geq-2\right\}$ do not form a subalgebra either.

(iii) In the limit $c \rightarrow \pm \infty$, the subset $w$ in (i) yields a closed, linear algebra, which is $S U(2,1)$.

(iv) In the non-chiral case, with left-handed and right-handed generators $L_{n}, W_{n}$ and $\bar{L}_{n}, \bar{W}_{n}$, and in the limit $c \rightarrow \pm \infty$, the set of generators $\left\{L_{0}-\bar{L}_{0}, L_{-1}, \bar{L}_{-1}\right.$, $\left.W_{0}-\bar{W}_{0}, W_{-2}, \bar{W}_{-2}, W_{-1}, \bar{W}_{-1}\right\}$ forms a subalgebra which one might call the $W_{3}$ Poincaré algebra.

(v) The classical (i.e., with Poisson brackets) version of the algebra without central charge reads

$$
\begin{aligned}
{\left[L_{m}, L_{n}\right] } & =(m-n) L_{m+n} \\
{\left[L_{m}, W_{n}\right] } & =(2 m-n) W_{m+n} \\
{\left[W_{m}, W_{n}\right] } & =(m-n)\left(\sum_{k} L_{m+n-k} L_{k}\right) .
\end{aligned}
$$

It is this algebra which is reproduced if one evaluates the commutator algebra of local symmetries under which the classical action of chiral $W_{3}$ gravity coupled to scalar matter fields is invariant [11]. The $(L L)$ term is then related to the appearance field-dependent structure functions in the commutator algebra. A classical $W_{3}$ algebra with central extension exists and was discussed in [12].

(vi) One can also take linear combintions of $W_{m}$ and $L_{m}$ and rescale such that if $c$ is a function of $\hbar$, all commutators vanish for $\hbar \rightarrow 0$ [13].

While the algebra (v) is related to the purely classical formulation, it has been found that the algebras (iii) and (iv) are related to (quanturn) induced $W_{3}$ gravity [5] and topological $W_{3}$ gravity [14].

\section{The classical formulations.}

At the classical level, one can consider covariant formulations of $W$ gravity theories, or consider these theories in special gauges such as the light-cone gauge or the chiral 
gauge (which is the light-cone gauge with only one chirality present).

In our approach to covariant $W_{3}$ gravity $[2,3]$, the gauge multiplet contains four vielbein fields $e_{\mu}^{+}, e_{\mu}^{-}$and four $W$-vielbein fields $\hat{b}_{\mu}{ }^{++}, b_{\mu}{ }^{--}$. The local gange invariances are: general coordinate, Weyl and local Lorentz symmetries, together with their $W$ analogues. By fixing some of the algebraic symmetries, one obtains the light-cone theory, and by fixing additional symmetries one obtains the chiral theory.

In the chiral theory there are only two local symmetries left: $\epsilon$ symmetry and $\lambda$ symmetry, with parameters $\epsilon_{+}$and $\lambda_{++}$. They arise as particular linear combinations of all local symmetries present in the covariant formulation, which are chosen such that the chiral gauge is preserved.

Let us now discuss the coupling of the $W_{3}$ gauge fields to scalar matter fields $\phi^{i}$, $i=1,2, \ldots, N$. We should first explain the observation made by Hull [11], that a free action for $N$ scalar fields admits a rigid $W_{3}$ symmetry. The chiral gauge transformations of the scalar fields read

$$
\delta_{\epsilon} \phi^{i}=\epsilon_{+} \partial_{-} \phi^{i}, \quad \delta_{\lambda} \phi^{i}=\lambda_{++} d^{i j k} \partial_{-} \phi^{j} \partial_{-} \phi^{k}
$$

where $d^{i j k}$ is a symmetric 3 -index tensor. [We will denote the complex coordinates of $d=2$ euclidean space-time by $z, \bar{z}$ and write $\partial_{-}$and $\partial_{+}$for $\partial_{z}$ and $\partial_{\bar{z}}$, respectively.] One can now promote these symmetries to local gauge invariances by introducing gauge fields $h_{++}$and $b_{+++}$in the standard way. It turns out [11] that the scalar field action with only the minimal coupling to these gauge fields,

$$
S_{\mathrm{ch}}=\frac{1}{\pi} \int d^{2} z\left[-\frac{1}{2} \partial_{+} \phi^{i} \partial_{-} \phi^{i}+\frac{1}{2} h_{++} \partial_{+} \phi^{i} \partial_{+} \phi^{i}+\frac{1}{3} b_{+++} d^{i j k} \partial_{+} \phi^{i} \partial_{+} \phi^{j} \partial_{+} \phi^{k}\right],
$$

is gauge-invariant, provided we choose the transformation rules of 1, and $b_{+++}$appropriately and we have the identity

$$
d^{k(i j} d^{l) m k}=\delta^{(i j} \delta^{l) m}
$$

In a next step, we consider the light-cone formulation, with both chiralities present. We introduce gauge fields $h_{ \pm \pm}$and $b_{ \pm \pm \pm}$corresponding to the local symmtries $\epsilon_{ \pm}$and $\lambda_{ \pm \pm}$. In [1] we found that the light-cone gauge action is non-polynomial in the spin-3 
gauge fields. This difficulty can be circumvented by introducing auxiliary fields $F_{+}{ }^{i}$ and $F_{-}{ }^{i}$, which will later play the role of so-called nested covariant derivatives. With these variables, the gauge-invariant action takes the following form [1]

$$
\begin{gathered}
S_{\mathrm{lc}}=\frac{-1}{\pi} \int d^{2} z e\left[-\frac{1}{2} \nabla_{+} \phi^{i} \nabla_{-} \phi^{i}-F_{+}{ }^{i} F_{-}{ }^{i}+F_{+}{ }^{i}\left(\nabla_{-} \phi^{i}-\frac{1}{3} b_{---} d^{i j k} F_{+}{ }^{j} F_{+}{ }^{k}\right)\right. \\
\left.+F_{-}{ }^{i}\left(\nabla_{+} \phi^{i}-\frac{1}{3} b_{-} \ldots d^{i j k} F_{-}{ }^{i} F_{-}{ }^{k}\right)\right]
\end{gathered}
$$

where $e=\left(1-h_{++} h_{--}\right)^{-1}$ and $\nabla_{ \pm}=\partial_{ \pm}-h_{ \pm \pm} \partial_{\mp}$.

The field equation of $F_{-}{ }^{i}$ is algebraic and leads to

$$
F_{+}{ }^{i}=\nabla_{+} \phi^{i}-b_{+++} d^{i j k} F_{-}{ }^{j} F_{-}{ }^{k}
$$

(with a similar result for $F_{-}{ }^{i}$ ). When solving $F_{ \pm}^{i}$ by iteration one obtains a generalization of a covariant derivative, which is infinitely nonlinear and is appropriately called a nested covariant derivative.

A variant of this light-cone formulation was later obtained in [15] where the spin-2 and spin-3 fields are treated symmetrically, leading to the action

$$
\begin{aligned}
S_{\mathrm{lc}}^{\prime}= & \frac{-1}{\pi} \int d^{2} z\left[-\frac{1}{2} \partial_{+} \phi^{i} \partial_{-} \phi^{i}-F_{+}{ }^{i} F_{-}{ }^{i}\right. \\
& \left.+\left\{F_{+}{ }^{i}\left(\partial_{-} \phi^{i}-\frac{1}{2} h_{--} F_{+}{ }^{i}-\frac{1}{3} b_{---} d^{i j k} F_{+}{ }^{j} F_{+}{ }^{k}\right)+(+\leftrightarrow-)\right\}\right] .
\end{aligned}
$$

Upon elimination of $F_{ \pm}{ }^{i}$, the results from (3.4) and (3.6) should be equivalent up to redefinitions; this has been checked to some orders in fields [15].

A covariant formulation of $W_{3}$ gravity ${ }^{8}$ can now be obtained by completing the gravitational covariantizations (giving for example $\nabla_{ \pm} \varphi^{i}=e_{ \pm}{ }^{\mu} \partial_{\mu} \varphi^{i}$ ) and by incorporating all four spin-3 fields $b_{+}{ }^{ \pm \pm} \equiv e_{+}{ }^{\mu} b_{\mu}{ }^{ \pm \pm}$and $b_{-}{ }^{ \pm \pm}$. The $F_{-}{ }^{i}$-field equations should now contain an extra term with $b_{+}{ }^{++}$according to

$$
F_{+}{ }^{i}-\nabla_{+} \phi^{i}+\left(b_{+}{ }^{++} F_{+}{ }^{j} F_{+}{ }^{k}+b_{+}{ }^{--} F_{-}{ }^{j} F_{-}{ }^{k}\right) d^{i j k}=0,
$$

\footnotetext{
${ }^{8}$ This covariant formulation was first derived in $[2,3]$, where we employed a gauge procedure based on the algebra (2.9). This procedure naturally explains the origin of all eight local symmetries and the (complicated) form of the trarisformation rules of the fields.
} 
and similarly for $F_{+}{ }^{i}$. However, these equations cannot directly be written as $\delta S / \delta F_{ \pm}^{i}=$ 0 , since that would violate the integrability conditions for these field equations. Instead, one may obtain the covariant action by putting

$$
\frac{\delta S}{\delta F_{ \pm}^{i}}=A_{i j}^{( \pm)}\left(F_{\mp}^{j}-\ldots\right)
$$

where the $A_{i j}{ }^{( \pm)}$are integrating factors and the dots are as in (3.7). One can then determine the $A_{i j}{ }^{( \pm)}$and, eventually, the action. The latter reads [2]

$$
\begin{aligned}
S_{\mathrm{cov}} & =\frac{-1}{\pi} \int d^{2} z e\left[-\frac{1}{2} \nabla_{+} \phi^{i} \nabla_{-} \phi^{i}-F_{+}{ }^{i} F_{-}{ }^{i}\right. \\
& +\left\{F_{+}{ }^{i}\left(\nabla_{-} \phi^{i}-\frac{1}{3} b_{-}{ }^{++} F_{+}{ }^{j} F_{+}{ }^{k} d^{i j k}\right)+(+\leftrightarrow-)\right\} \\
& \left.+\left\{-b_{+}{ }^{++} d^{i j k} F_{+}{ }^{i} F_{+}{ }^{j}\left(F_{-}{ }^{k}-\nabla_{-} \phi^{k}+\frac{1}{2} b_{-}{ }^{ \pm \pm} d^{k l m} F_{ \pm}{ }^{\ell} F_{ \pm}{ }^{m}\right)+(+\leftrightarrow-)\right\}\right]
\end{aligned}
$$

(in the last term we sum over the two combinations of indices). Some classical field equations of particular interest are

$$
\begin{aligned}
T_{++}(F) \equiv-\frac{1}{2} F_{+}{ }^{i} F_{+}{ }^{i} & =0, & W_{+++}(F) & \equiv-\frac{1}{3} d^{i j k} F_{+}{ }^{i} F_{+}{ }^{j} F_{+}{ }^{k}=0 \\
T_{--}(F) & =0, & W_{---}(F) & =0 .
\end{aligned}
$$

Let us remark that it is not yet clear that this particular 'covariant' formulation of $W_{3}$ gravity will turn out to be the most convenient for studying, for example, global characteristics of $W_{3}$ gravity. One also might consider a 'metric formulation', in terms of rank-2 and 3 completely symmetric tensors. The distinction, which is largely irrelevant at the classical level, might have some important consequences at the quantum level.

Apart from $W_{3}$ gravity, one can consider gravity theories associated to more general $W$ algebras such as $W_{4}, W_{5}$, etc. and also $w_{\infty}$ and $W_{\infty}[15,16,17]$. The latter theories contain spins ranging up to infinity. One may therefore consider the spin label as a Fourier index, in which case the $w_{\infty}\left(W_{\infty}\right)$ theories might correspond to some, as yet unknown, gauge theory in two complex (or four real) dimensions, perhaps describing self-dual gravitational instantons. The $w_{\infty}$ algebra reads

$$
\left[t_{m}{ }^{j}, t_{n}{ }^{k}\right]=[(j-1) n-(k-1) m] t_{m+n}^{j+k-2}
$$


with $j, k \geq 2$ and $m \geq-j+1, n \geq-k+1$, and is clearly a linear algebra. Various aspects of $W_{\infty}$ are discussed in [18].

Let us finally mention that $W_{3}$ supergravities exist, both for the chiral theory [11] and for the light-cone theory [19]. A covariant $W$ supergravity has not yet been found.

\section{Quantum aspects.}

In the analysis of quantum $W_{3}$ gravity one can distinguish two approaches: the critical approach and the noncritical approach.

In the critical approach, one chooses a matter system and tries to construct an anomaly-free coupling to the $W_{3}$ gravity degrees of freedom. In particular, one can choose a matter system consisting of scalar fields, and study the theory in a Lagrange formulation. Upon quantization, one would then add the usual Faddeev-Popov ghosts to the action, and try to cancel all gauge anomalies by adding suitable counter terms to the quantum action. These extra terms will necessarily include so-called background couplings, which contain second or higher derivatives of the scalar fields $\phi^{i}$. This can be seen as follows.

If one evaluates the 1 and 2 loop anomalies in the presence of ghosts $[20,21,22$, 23 ], one finds that all individual anomalies (including the so-called matter dependent anomalies proportional to $\left.\partial_{-} \phi \partial_{-}^{3} \phi\right)$ either vanish or can be cancelled by adding a certain matter independent counterterm to the quantum action. However, the simultaneous cancellation of all anomalies is only possible if, in addition, one adds certain matter dependent counterterms to the quantum action $[24,22,25]$. The easiest way to obtain the full, anomaly-free quantum action is by using the form of the quantum BRST charge for $W_{3}$, which has been known since 1987 [26]. Such a nilpotent BRST charge only exists if the matter system involved has exact $W_{3}$ symmetry at the quantum level. This then makes clear the origin of the background couplings, since it is known [27] that the realization of $W_{3}$ currents in terms of scalar fields necessar:iy (for $c \neq 2$ ) involves the introduction of background charges. The complete results for the anomaly-free coupling were first presented in [28].

In the noncritical approach (pioneered for ordinary gravity by Polyakov [29], KPZ [30] and Al.B. Zamolodchikov [31]) one does not try to cancel the anomalies, but, on the contrary, one keeps them in the theory, and uses the fact that they make the spin 2 
and spin 3 gauge fields propagating at the quantum level. In the critical approach the effective action vanishes (it is proportional to $c-100$, just like the effective action of gravity is proportional to $c-26$ and in order that the anomalies cancel, c must be 100 in $W_{3}$ gravity), but in the noncritical approach the effective action is nonvanishing.

In the noncritical approach, one can distinguish between the induced action (obtained by integrating only over the matter system), and the effective action (obtained by integrating also over the gauge fields). One does not add ghosts, nor gauge-fixing terms, for the anomalous gauge symmetries. So far, only the chiral approach has been studied. The induced action is defined by

$$
e^{\Gamma_{\text {ind }}[h, b ; c]}=\left\langle e^{-\frac{1}{\pi} \int d^{2} z(h T+b W)}\right\rangle
$$

where $T$ and $W$ are abstract currents, satisfying the operator product expansions (OPE) of the $W_{3}$ algebra in (2.8). The simplest example would be to use two scalar fields, since

$$
T=-\frac{1}{2} \partial_{-} \phi^{i} \partial_{-} \phi^{i}, \quad W=\frac{-i}{6} d^{i j k} \partial_{-} \phi^{i} \partial_{-} \phi^{j} \partial_{-} \phi^{k},
$$

with $d^{000}=1, d^{011}=-1$, form an exact $W_{3}$ algebra with $c=2$. (Note that the field $b$ in (4.1) has been rescaled by a factor of $i / 2$ as compared to the field $b$ in (3.2).) For a general number $n$ of scalar fields, $W_{3}$ currents with adjustable central charge, can be constructed by using background charge couplings [27].

The OPE's for the $W_{3}$ algebra were given in section 2. Using that $\langle T\rangle=\langle W\rangle=0$, one can compute $\Gamma_{\text {ind }}[h, b ; c]$ order-by-order in perturbation theory. We shall write down two exact Ward identities (anomalous current conservation laws) for $\Gamma_{\text {ind }}[h, b ; c]$, which are nonlocal and nonlinear. The first nonlocalities appear at the 3-loop level; we will compute them explicitly. We will see that these terms mark the onset of a $1 / c$ expansion: whereas up to three loops all terms in $\Gamma_{\text {ind }}[h, b ; c]$ are proportional to $c$, one finds that from the 3-loop level on there are also terms proportional to $c^{0}, c^{-1}$, etc . In the limit $c \rightarrow \pm \infty$, one obtains a local (but still nonlinear) Ward identity for the induced action.

The effective action is obtained by taking the Legendre transform of the generating 
functional $W[t, w ; c]$ for connected graphs

$$
\Gamma_{\mathrm{eff}}[h, b ; c]=W[t, w ; c]-\frac{1}{\pi} \int d^{2} z(h t+b w)
$$

with

$$
\pi \frac{\delta W}{\delta t}=h, \quad \pi \frac{\delta W}{\delta w}=b
$$

The latter is defined by

$$
e^{W[t, w ; c]}=\int d h d b e^{\Gamma_{\mathrm{ind}}[h, b ; c]+\frac{1}{\pi} \int d^{2} z(h t+b w)} .
$$

The main results obtained in $[5,6]$, and which we shall discuss below, can be summarized as follows.

(i) $\Gamma_{\text {ind }}[h, b ; c \rightarrow \pm \infty]$ can be obtained in closed form by 'reduction' (i.e., imposing constraints, which can actually be solved) of the induced action for an $\operatorname{Sl}(3, \mathbf{R})$ gauge theory coupled to matter.

(ii) The Ward identity for $\Gamma_{\text {eff }}[h, b ; c]$ becomes local, since the nonlocalities of $\Gamma_{\text {ind }}[h, b ; c]$ (which start at level $c^{\circ}$ ) are canceled by nonlocalities coming from $h$ and $b$ loops.

(iii) $\Gamma_{\text {eff }}[h, b ; c]$ is obtained by (finitely) renormalizing $\Gamma_{\text {ind }}[h, b ; c \rightarrow \pm \infty]$,

$$
\Gamma_{\mathrm{eff}}[h, b ; c]=Z_{\mathrm{c}} \Gamma_{\mathrm{ind}}\left[Z_{h} h, Z_{b} b, c \rightarrow \pm \infty\right]
$$

(iv) By choosing suitable variables other than $h, b$, the effective action itself (and not just the defining Ward identity) becomes local. [For pure gravity, this was already found to be the case by Polyakov [29], who introduced a new variable $f$, related to $h_{++}$by $h_{++}=\partial_{+} f /\left(\partial_{-} f\right)$. So our new variables extend Polyakov's $f$ variable to the case of $W_{3}$ gravity.]

We finally comment on the tentative interpretation of scalar field theories coupled to $W_{3}$ gravity as $W_{3}$ strings $[32,33,34]$. Depending on the formulation, these would be 
critical or non-critical $W_{3}$ strings, respectively. However, the critical $W_{3}$ strings have the unusual (as compared to bosonic or supersymmetric strings) feature of the background charges that we discussed above. An interesting possibility would be that the two scalar fields that acquire background charges can be interpreted as remnants of $W_{3}$ gauge field degrees of freedom, which could lead to an equivalence with the formulation as noncritical $W_{3}$ strings. (This point of view is clearly supported by the results of [33].) This possibility, and other aspects of $W_{3}$ strings, are presently under study.

In the remaining sections we will present in some detail our derivations leading to the results (i) to (iv). Some of these derivations have not appeared elsewhere.

\section{The Ward iclentities for the induced action.}

To obtain the Ward identities for the induced action of chiral $W_{3}$ gravity, which we shall from now on denote by $\Gamma_{\text {ind }}^{W_{3}}[h, b]$ instead of $\Gamma_{\text {ind }}[h, b ; c]$,

$$
e^{\Gamma_{\mathrm{ind}}^{W_{3}}[h, b]}=\left\langle e^{S_{\mathrm{int}}}\right\rangle, \quad S_{\mathrm{int}}=-\frac{1}{\pi} \int d^{2} z(h T+b W),
$$

we begin by varying $h$ under $\epsilon$ symmetry as $\delta h=\partial_{+} \epsilon$, and $b$ under $\lambda$ symmetry as $\delta b=\partial_{+} \lambda$. Then we cancel any $\beta$-independent variation proportional to $T$ or $W$ by adding suitable extra terms in $\delta h$ and $\delta b$, respectively. The left-over is then the anomaly of the induced action under these $\delta h$ and $\delta b$ variation rules, and the Ward identity is obtained by removing the gauge parameter from the equation $\delta \hbar \frac{\delta}{\delta h} \Gamma_{\text {ind }}^{W_{3}}+\delta b \frac{\delta}{\delta b} \Gamma_{\text {ind }}^{W_{3}}=$ anomaly.

We begin with the easier case, the $\epsilon$ symmetry. Under $\delta h=\partial_{+} \epsilon$, the induced action varies as follows

$$
\left(\delta \Gamma_{\text {ind }}^{W_{3}}\right) \exp \Gamma_{\text {ind }}^{W_{3}}=\left\langle\left(\frac{-1}{\pi} \int \partial_{+} \epsilon T d^{2} z\right) e^{S_{\text {int }}}\right\rangle .
$$

The vacuum expectation value of a product of $T(z)$ with other operators $A_{1}\left(z_{1}\right) \ldots A_{n}\left(z_{n}\right)$ can be written, according to BPZ [35], as multiple contractions. ${ }^{9}$ For example

$$
\left\langle T(z) A\left(z_{1}\right) . . A\left(Z_{2}\right)\right\rangle=\left\langle T(z) A\left(z_{1}\right) A\left(z_{2}\right)\right\rangle+\langle A\left(z_{1}\right) T \underbrace{(z) A\left(z_{2}\right)}\rangle .
$$

\footnotetext{
${ }^{9}$ On-shell the component $T(z, \bar{z})=T_{--}(z, \bar{z})$ does not depend on $\bar{z}$. In the OPE's only the on-shell part contributes and we shall write $T(z)$.
} 
[This property can be derived by considering the behaviour under $z \rightarrow z+\epsilon(z)$ of the correlator $\left\langle A_{1}\left(z_{1}\right) \ldots A_{n}\left(z_{n}\right)\right\rangle$.]

It follows that

$$
\left(\delta \Gamma_{\text {ind }}^{W_{3}}\right) \exp \Gamma_{\text {ind }}^{W_{3}}=\left\langle\left(-\frac{1}{\pi} \int\left(\partial_{+} \epsilon\right) T d^{2} z_{1}\right)\left(-\frac{1}{\pi} \int(h T+b W) d^{2} z_{2}\right) e^{S_{\text {int }}}\right\rangle
$$

where the hooks indicate that one should take the singular terms in the $T T$ and $T W$ OPE's. From the TT OPE we obtain

$$
\left\langle\frac{1}{\pi^{2}} \int \partial_{+} \epsilon\left(z_{1}\right) h\left(z_{2}\right)\left[\frac{c / 2}{\left(z_{1}-z_{2}\right)^{4}}+\frac{2 T\left(z_{2}\right)}{\left(z_{1}-z_{2}\right)^{2}}+\frac{T^{\prime}\left(z_{2}\right)}{\left(z_{1}-z_{2}\right)}\right] d^{2} z_{1} d^{2} z_{2} e^{S_{\text {int }}}\right\rangle .
$$

We now use the following identity for distributions

$$
\frac{1}{\left(z_{1}-z_{2}\right)^{n}}=\pi \frac{(-1)^{n+1}}{(n-1) !} \frac{\partial_{z_{1}}^{n-1}}{\partial_{\bar{z}_{1}}} \delta^{2}\left(z_{1}-z_{2}\right) \text {. }
$$

[It can be proven by integrating $z_{2}$ over the plane minus a small disk around $z_{1}$. Multiplying both sides with $\partial_{\bar{z}_{1}}$, one obtains on the left-hand side the derivative of the $\theta$ function $\theta\left[\left(z_{1}-z_{2}\right)\left(\bar{z}_{1}-\bar{z}_{2}\right)-\epsilon^{2}\right]$, which is proportional to a delta function.] Using this identity we find

$$
\begin{gathered}
\left(\delta \Gamma_{\text {ind }}^{W_{3}}\right) \exp \Gamma_{\text {ind }}^{W_{3}}=\langle \\
\left\langle\left[\left(\frac{-c}{12 \pi} \int \partial_{-}^{3} \epsilon h d^{2} z\right)+\left(-\frac{1}{\pi} \int 2 \partial_{-} \epsilon h T d^{2} z\right)\right.\right. \\
\left.+\left(-\frac{1}{\pi} \int \epsilon h \partial_{-} T^{\prime} d^{2} z\right)\right] e^{\left.S_{\text {int }}\right\rangle}
\end{gathered}
$$

All terms with $T$ can be canceled by adding two suitable extra terms to $\delta h$

$$
\delta h=\partial_{+} \epsilon+\epsilon \partial_{-} h-h \partial_{-} \epsilon .
$$

We are then left with 'the minimal anomaly' containing the $h$ field. If the sources $T$ and $W$ are realized by a local lagrangian scalar field theory as in (4.2), the Feynman diagram which yields this minimal anomaly is given by

$$
\delta_{0} \sim 0 \sim \sim=-\frac{c}{12 \pi} \int h \partial_{-}^{3} \epsilon d^{2} z
$$


We still have to deal with the $T W$ contractions in (5.4). They yield

$$
\begin{aligned}
& \left\langle\left(\frac{1}{\pi^{2}} \int \partial_{+} \epsilon\left(z_{1}\right) b\left(z_{2}\right)\left[\frac{3 W\left(z_{2}\right)}{\left(z_{1}-z_{2}\right)^{2}}+\frac{W^{\prime}\left(z_{2}\right)}{\left(z_{1}-z_{2}\right)}\right] d^{2} z_{1} d^{2} z_{2}\right) e^{S_{\text {int }}}\right\rangle \\
& =\left\langle-\frac{1}{\pi} \int\left(3 \partial_{-} \epsilon b W+\epsilon b W^{\prime}\right) d^{2} z e^{S_{\text {int }}}\right\rangle .
\end{aligned}
$$

All these variations are canceled by adding suitable terms to $\delta b$

$$
\delta b=\epsilon \partial_{-} b-2 b \partial_{-} \epsilon \text {. }
$$

Defining variables $u$ and $v$ proportional to the effective currents

$$
T_{\mathrm{eff}}=-\pi \frac{\delta \Gamma_{\mathrm{ind}}^{W_{3}}}{\delta h} \equiv \frac{c}{12} u, \quad W_{\mathrm{eff}}=-\pi \frac{\delta \Gamma_{\mathrm{ind}}^{W_{3}}}{\delta b} \equiv \frac{c}{360} v
$$

so that

$$
u=\frac{\partial_{-}^{3}}{\partial_{+}} h+\ldots, \quad v=\frac{\partial_{-}^{5}}{\partial_{+}} b+\ldots,
$$

the final Ward identity corresponding to $\epsilon$ symmetry for the induced action reads

$$
\partial_{+} u=D_{1} h+\frac{1}{30}\left(3 v \partial_{-}+2 \partial_{-} v\right) b
$$

where

$$
D_{1} \equiv \partial_{-}^{3}+2 u \partial_{-}+u^{\prime}
$$

This is a (complicated) current conservation law $\left(\partial_{+} u \sim \partial^{\mu} T_{\mu_{-}}\right)$with an anomaly $\left(\partial_{-}^{3} h\right)$. It is suggestive to consider all terms except the anomaly to constitute a covariant derivative on the doublet of currents $u$ and $v$, but at present no geometry is known which can explain this. We observe that this Ward identity is local: the non-local operator $\frac{1}{\partial_{+}}$does not appear explicitly but is hidden inside the currents $u$ and $v$.

We now turn to the $\lambda$ symmetry. Here, the nonlinearities in the OPE of $W$ with itself are expected to lead to interesting complications. We begin as in the case of $\epsilon$ symmetry, and vary $b$ as $\delta b=\partial_{+} \lambda$. This yields

$$
\left(\delta \Gamma_{\text {ind }}^{W_{3}}\right) \exp \Gamma_{\text {ind }}^{W_{3}}=\left\langle\left(-\frac{1}{\pi} \int \partial_{+} \lambda W d^{2} z_{1}\right)\left(-\frac{1}{\pi} \int(h T+b W) d^{2} z_{2}\right) e^{S_{\text {int }}}\right\rangle
$$


The $W T$ OPE yields terms with a $W$, namely $3 W\left(z_{2}\right) /\left(z_{1}-z_{2}\right)^{2}+2 W^{\prime}\left(z_{2}\right) /\left(z_{1}-z_{2}\right)$, which are treated with (5.6) and are canceled by a suitable $b$ transformation law

$$
\delta_{\lambda} b=\partial_{+} \lambda+2 \lambda \partial_{-} h-h \partial_{-} \lambda
$$

From the $W W$ contraction we get a central charge term, terms linear in $T$ and terms with $\Lambda$. The central charge term $(c / 3)\left(z_{1}-z_{2}\right)^{-6}$ yields the minimal $\lambda$ anomaly proportional to $\partial_{-}^{5} b$, which is the counterpart of the minimal $\epsilon$ anomaly proportional to $\partial_{-}^{3} h$. The terms linear in $T$ are all canceled by a suitable law for $h$

$$
\delta_{\lambda} h=\frac{1}{30}\left(2 \lambda \partial_{-}^{3} b-3 \partial_{-} \lambda \partial_{-}^{2} b+3 \partial_{-}^{2} \lambda \partial_{-} b-2 \partial_{-}^{3} \lambda b\right) .
$$

The remaining terms with $\Lambda$ appear in $\delta_{\lambda} \Gamma_{\text {ind }}^{W_{3}}$ as $\left\langle\Lambda(z) \exp S_{\text {int }}\right\rangle \equiv \Lambda_{\text {eff }}(z)$, and will be studied in more detail in the next section. Thus the response of $\Gamma_{\text {ind }}^{W_{3}}$ under $\delta_{\lambda} h$ and $\delta_{\lambda} b$ given above contains the minimal anomaly and the terms with $\Lambda_{\text {eff. }}$. The corresponding $\lambda$ Ward identity reads

$$
\partial_{+} v=D_{2}^{\text {lin }} b+\left(3 v \partial_{-}+\partial_{-} v\right) h+\frac{360}{c} \beta\left(b \partial_{-}+2 \partial_{-} b\right) \Lambda_{\mathrm{eff}}
$$

with

$$
D_{2}^{l i n}=\partial_{-}^{5}+\left(10 u \partial_{-}^{3}+15 u^{\prime} \partial_{-}^{2}+9 u^{\prime \prime} \partial_{-}+2 u^{\prime \prime \prime}\right)
$$

and $\beta$ as in (2.3).

In the next section we shall show that all $h$-dependent but $b$-independent terms in $\Lambda_{\text {eff }}$ are local when written in terms of $u$, and can be transferred to $D_{2}^{\text {lin }}$, which then becomes the Gelfand-Dickey operator $D_{2}$

$$
D_{2}=D_{2}^{l i n}+16 u u^{\prime}+16 u^{2} \partial_{-} .
$$

The $b^{2}$ terms in $\Lambda_{\text {eff }}$, however, can not all be written as local expressions in terms of the variables $h, b, u, v$, and they lead to nonlocal $b^{3}$ terms in the $\lambda$ Ward identity. The exact form of these $b^{3}$ terms is not an easy matter to obtain, but we shall need it in order to later show that they cancel against other nonlocal terms due to integrating 
the induced action over $h$ and $b$. A simple dimensional argument already shows that nonlocalities must appear in the $\lambda$ Ward identity. (By dimension we mean the usual notion according to which a coordinate has dimension -1 . In conformal field theory this corresponds to the sum of left and right conformal dimensions.) To see this, note that $\Lambda_{\text {eff }}$ has dimension $4, h$ has dimension $0, b$ has dimension $-1, u$ has dimension 2 , while $v$ has dimension 3. The $h^{2}$ terms in $\Lambda_{\text {eff }}$ may appear as $u^{2}$ as, in fact, they do (see (6.7)), but the $b^{2}$ terms cannot be produced by a local expression in $h, b, u, v[v$ is odd in $b$, hence $b^{2} v^{2}$ and $\partial_{-} v$ are out, while buv produces at best an $h b^{2}$ term but no $b^{2}$ term. Further, $\partial_{-}^{2} u$ is out because it would also produce a term with a simple $h$ field, whereas $\Lambda_{\text {eff }}$ contains no terms linear in $h$ or $b$. Finally, a term with $\partial_{-}^{2} b v$ is out, as the dual requirements of dimensions and Lorentz covariance (the correct left- and right-conformal dimensions) imply nonlocality]. Thus, nonlocalities in $\Lambda_{\text {eff }}$ cannot be avoided.

\section{Computing $\Lambda_{\mathrm{eff}}(h, b)$.}

We now study the function $\Lambda_{\text {eff }}(h, b)$ in more detail. From its definition

$$
\begin{aligned}
\Lambda_{\mathrm{eff}} & =\left\langle\Lambda(z) \exp -\frac{1}{\pi} \int(h T+b W) d^{2} z_{1}\right\rangle, \\
\Lambda(z) & =(T T)(z)-\frac{3}{10} T^{\prime \prime}(z)
\end{aligned}
$$

we see that we shall need the singular terms in the OPE of $\Lambda(z)$ with $T\left(z_{1}\right)$ aind $W\left(z_{1}\right)$. As we shall discuss in a moment, the first result is (compare with (2.6))

$$
T\left(z_{1}\right) \Lambda(z)=\frac{5 c+22}{5} \frac{T(z)}{\left(z_{1}-z\right)^{4}}+\left(\frac{4}{\left(z_{1}-z\right)^{2}}+\frac{\partial_{z}}{z_{1}-z}\right) \Lambda(z) .
$$

Were it not for the term with $T$ on the right hand side, $\Lambda$ would be a primary field with dimension 4. For the contraction of $\Lambda(z)$ with $W\left(z_{1}\right)$ one finds

$$
\begin{aligned}
W\left(z_{1}\right) \Lambda(z)= & \left\{\frac{6(T W)(z)}{\left(z_{1}-z\right)^{2}}+\frac{4\left(T W^{\prime}\right)\left(z^{\prime}\right)}{\left(z_{1}-z\right)}+\frac{15 W(z)}{\left(z_{1}-z\right)^{4}}+\frac{8 W^{\prime}(z)}{\left(z_{1}-z\right)^{3}}+\frac{\frac{3}{2} W^{\prime \prime}(z)}{\left(z_{1}-z\right)^{2}}\right\} \\
& -\frac{3}{10}\left\{\frac{18 W(z)}{\left(z_{1}-z\right)^{4}}+\frac{16 W^{\prime}(z)}{\left(z_{1}-z\right)^{3}}+\frac{7 W^{\prime \prime}(z)}{\left(z_{1}-z\right)^{2}}+\frac{2 W^{\prime \prime \prime}(z)}{\left(z_{1}-z\right)}\right\} .
\end{aligned}
$$


We have given the contractions of $W\left(z_{1}\right)$ with $(T T)(z)$ and $T^{\prime \prime}(z)$ separately (compare with (6.1)).

We now restrict our attention to the terms in $\Lambda_{\text {eff }}$ with only one or two $h$ and/or $b$ fields. There are no terms with zero or one field, as in the expansion

$$
\Lambda_{\mathrm{eff}}=\left\langle\Lambda(z)+\Lambda(z)\left(-\frac{1}{\pi} \int(h T+b W) d^{2} z\right)+\ldots\right\rangle
$$

the terms explicitly written vanish due to $\langle\Lambda\rangle=0,\langle T\rangle=0$ and $\langle W\rangle=0$. The contributions to $\Lambda_{\text {eff }}$ with two fields are given by

$$
\left\langle\Lambda(z) \frac{1}{2 !}\left(-\frac{1}{\pi} \int(h T+b W) d^{2} z_{1}\right)\left(-\frac{1}{\pi} \int(h T+b W) d^{2} z_{2}\right)\right\rangle .
$$

We shall evaluate these correlators by putting $T\left(z_{1}\right)$ and $W\left(z_{1}\right)$ in front. Cross terms with $h b$ vanish since $\langle\Lambda T W\rangle$ vanishes.

The $h^{2}$ terms come from the contractions

$$
\frac{1}{2}\left\langle T\left(z_{1}\right) \Lambda(z) T\left(z_{2}\right)\right\rangle+\frac{1}{2}\langle\Lambda(z) T \underbrace{\left(z_{1}\right) T}\left(z_{2}\right)\rangle
$$

where only the $T \Lambda \sim T$ in the first term contributes, while the second term vanishes. It yields

$$
\begin{aligned}
\Lambda_{\mathrm{ef}}\left(h^{2}\right) & =\frac{5 c+22}{10} \int \frac{1}{\left(z_{1}-z\right)^{4}} \frac{c / 2}{\left(z-z_{2}\right)^{4}} h\left(z_{1}\right) h\left(z_{2}\right) d^{2} z_{1} d^{2} z_{2} \\
& =\frac{(5 c+22) c}{720}\left[\frac{\partial_{-}^{3}}{\partial_{+}} h(z)\right]^{2}=\frac{c}{45 \beta} u^{2}+\mathcal{O}\left(h^{3}\right)
\end{aligned}
$$

since the effective field equation $u$ is given by $u=\frac{\partial^{3}}{\partial_{+}} h+\ldots$. Thus we see that due to the fact that we can hide all nonlocalities of $\left[\frac{\partial_{-}^{3}}{\partial_{+}} h\right]^{2}$ in $u^{2}$ to the order in $h$ we work, the $h^{2}$ terms of $\Lambda_{\text {eff }}$ do not produce nonlocalities in the Ward identity. It can be shown [4] that the relation $\Lambda_{\mathrm{eff}}(h, b=0)=\frac{c}{45 \beta} u^{2}(h, b=0)$ is exact to all orders in $h$.

The situation with the $b^{2}$ terms is more complicated. Now we must evaluate

$$
\frac{1}{2}\left\langle W\left(z_{1}\right) \Lambda(z) W\left(z_{2}\right)\right\rangle+\frac{1}{2}\left\langle\Lambda(z) W\left(z_{1}\right) W\left(z_{2}\right)\right\rangle
$$


and all the $T W$ and $W$ terms in $W \Lambda$ contribute. From $W W$, however, we only need the terms with $\Lambda$. To evaluate $\left\langle\Lambda\left(z_{1}\right) \Lambda\left(z_{2}\right)\right\rangle$ we replace $\Lambda\left(z_{2}\right)$ by $(T T)\left(z_{2}\right)$ and use the result for $\Lambda(z) T\left(z_{2}\right)$. The final result for the $b^{2}$ terms in $\Lambda_{\text {eff }}$ reads

$$
\Lambda_{\mathrm{eff}}\left(b^{2}\right)=\frac{8 c}{5 \cdot 7 !} \frac{1}{\partial_{+}}\left[b \frac{\partial_{-}^{8}}{\partial_{+}} b+6 \partial_{-} b \frac{\partial_{-}^{7}}{\partial_{+}} b+14 \partial_{-}^{2} b \frac{\partial_{-}^{6}}{\partial_{+}} b+14 \partial_{-}^{3} b \frac{\partial_{-}^{5}}{\partial_{+}} b\right]
$$

As we already mentioned, for the $h^{2}$ terms in $\Lambda_{\text {eff }}$ we find the square of (the leading part of) the $h$ effective field equation (namely $u=\frac{\partial^{3}}{\partial_{+}} h+\ldots$ ). This came about because in the OPE only one pole structure contributed, namely $\left(z-z_{1}\right)^{-4}\left(z-z_{2}\right)^{-4}$, which factorized into $u^{2}=\left[\frac{\partial^{3}}{\partial_{+}} h\right]^{2}+\ldots$ For the $b^{2}$ terms several pole structures contribute. Those coming from $W\left(z_{1}\right) \Lambda(z) W\left(z_{2}\right)$ give terms with $\left(z_{1}-z\right)^{-p}\left(z-z_{2}\right)^{-q}$ with $p+q=10$ and $p=1,2,3,4$, while from $\Lambda(z) W\left(z_{1}\right) W\left(z_{2}\right)$ one obtains terms with $\left(z_{1}-z_{2}\right)^{-2}\left(z-z_{2}\right)^{-8}$ and $\left(z_{1}-z_{2}\right)^{-1}\left(z-z_{2}\right)^{-9}$. The former produce products of the form $\left[\frac{\partial_{-}^{p-1}}{\partial_{+}} b\right]\left[\frac{\partial^{q-1}}{\partial_{+}} b\right]$, while the latter yield structures of the form $\frac{\partial^{8}}{\partial_{+}}\left[b \frac{1}{\partial_{+}} b\right]$ and $\frac{\partial^{7}}{\partial_{+}}\left[b \frac{\partial_{-}}{\partial_{+}} b\right]$. One should be careful with such nonlocal expressions, as they can be rewritten in various other forms which look very different. For example, if we would have started to evaluate the OPE with $\Lambda(z)$ in front, and computed the three point function $\langle\Lambda W W\rangle$ as $\left\langle\Lambda(z) W\left(z_{1}\right) W\left(z_{2}\right)\right\rangle+\left\langle W\left(z_{1}\right) \Lambda(z) W\left(z_{2}\right)\right\rangle$, we would have found a sum of terms of the form $\frac{\partial^{\bullet}}{\partial_{+}}\left[b \frac{\partial^{t}}{\partial_{+}} b\right]$ with $s+t=8, s=0,1,2,3$. This is the result given in (6.9). To show that both results are equivalent, one could multiply the first result by $\frac{1}{\partial_{+}} \partial_{+}$, and act with $\partial_{+}$to the right.

Let us finally show how one goes about to compute the (singular) OPE of elementary fields with composites such as $\Lambda(z)$. As an example, we will show how to derive the OPE $T \Lambda$ given in (6.2).

We begin by recalling that the normal ordering of two operators $A$ and $B$ can in general be written as [36]

$$
(A B)(z)=\frac{1}{2 \pi i} \oint \frac{d x}{x-z} A(x) B(z)
$$

where the $x$-contour runs around $z$. For contractions of operators with such composites, 
there is a Wick theorem [36], which in our case can be written as

$$
T \underbrace{(z)(T T)}(w)=\frac{1}{\pi} \int \frac{d x}{x-w}[T(z) T(x) T(w)+T(x) T(z) T(w)] .
$$

Substituting the $T \Gamma$ OPE one obtains

$$
\begin{aligned}
& \frac{1}{2 \pi i} \oint \frac{d x}{x-w}\left[\frac{c / 2}{(z-x)^{4}}+\frac{2 T(x)}{(z-x)^{2}}+\frac{T^{\prime}(x)}{z-x}\right] T(w) \\
& +\frac{1}{2 \pi i} \oint \frac{d x}{x-w} T(x)\left[\frac{c / 2}{(z-w)^{4}}+\frac{2 T(w)}{(z-w)^{2}}+\frac{T^{\prime}(w)}{z-w}\right]
\end{aligned}
$$

The second line gives straightaway

$$
\frac{(c / 2) T(w)}{(z-w)^{4}}+\frac{2(T T)(w)}{z-w)^{2}}+\frac{\left(T T^{\prime}\right)}{z-w}
$$

but in the first line we must expand $(z-x)^{-2}$ and $(z-x)^{-1}$ in $x$ about $w$ since any positive powers of $(x-w)$ can be overcome by the singularities in the OPE of $T(x) T(w)$. The leading terms of the first line give

$$
\frac{(c / 2) T(w)}{(z-w)^{4}}+\frac{2(T T)(w)}{(z-w)^{2}}+\frac{\left(T^{\prime} T\right)(w)}{z-w}
$$

while the terms obtained by expanding the poles yield

$$
\frac{3 c}{(z-w)^{6}}+\frac{8 T(w)}{(z-w)^{4}}+\frac{3 T^{\prime}(w)}{(z-w)^{3}}
$$

One can cancel the central term by adding to $(T T)(w)$ a term proportional to $T^{\prime \prime}(w)$. Indeed,

$$
T(z) T^{\prime \prime}(w)=\frac{10 c}{(z-w)^{6}}+\frac{12 T(w)}{(z-w)^{4}}+\frac{10 T^{\prime}(w)}{(z-w)^{3}}+\frac{4 T^{\prime \prime}}{(z-w)^{2}}+\frac{T^{\prime \prime \prime}(w)}{z-w}+\ldots
$$

so that $\Lambda=(T T)-\frac{3}{10} T^{\prime \prime}$ has an OPE with $T$ without central term. It was given in (6.2). 


\section{The $b^{4}$ terms in the induced action.}

The Ward identities for the induced action can be expanded in terms of $1 / c$, and their solution can also be expanded in a $1 / c$ series. The Ward identities read (see (5.14) and $(5.19))$

$$
\begin{aligned}
& \partial_{+} u=D_{1} h+\frac{1}{30}\left(3 v \partial_{-}+2 v^{\prime}\right) b \\
& \partial_{+} v=D_{2} b+\left(3 v \partial_{-}+v^{\prime}\right) h+\frac{360}{c} \beta\left(b \partial_{-}+2 b^{\prime}\right)\left(\Lambda_{\mathrm{eff}}-\frac{c}{45 \beta} u^{2}\right)
\end{aligned}
$$

where

$$
\Lambda_{\mathrm{eff}}-\frac{c}{45 \beta} u^{2}=\frac{8 c}{5 \cdot 7 !} \frac{1}{\partial_{+}} Q_{b b}^{8}+\mathcal{O}\left(b^{2} h^{\geq 1}\right)+\mathcal{O}\left(b^{4} h^{\geq 0}\right) \ldots
$$

with

$$
Q_{b b}^{8}=b \frac{\partial_{-}^{8}}{\partial_{+}} b+6 \partial_{-} b \frac{\partial_{-}^{7}}{\partial_{+}} b+14 \partial_{-}^{2} b \frac{\partial_{-}^{6}}{\partial_{+}} b+14 \partial_{-}^{3} b \frac{\partial_{-}^{5}}{\partial_{+}} b .
$$

The contributions due to $\Lambda_{\mathrm{eff}}-\frac{c}{45 \beta} u^{2}$ are all suppressed by at least one factor $1 / c$ as compared to the pure $u$ terms in $\Lambda_{\text {eff }}$. For example, in $\Lambda T T$ each contraction can give a factor $c$, but in $\Lambda W W$ the $\Lambda W$ contraction is of order $c^{\circ}$. The leading terms in $1 / c$ in the Ward identity, namely, the $c$-independent terms, read therefore

$$
\begin{aligned}
& \partial_{+} u=D_{1} h+\frac{1}{30}\left(3 v \partial_{-}+2 v^{\prime}\right) b \\
& \partial_{+} v=D_{2} b+\left(3 v \partial_{-}+v^{\prime}\right) h .
\end{aligned}
$$

These equations generalize the KdV equations, and are called the Boussinesq equations. They characterize the induced action in the limit $c \rightarrow \pm \infty$. We have solved these coupled nonlinear partial differential equations for $u$ and $v$ in terms of $h$ and $b$. The solution is

$$
u_{L}(h, b)=-\pi \frac{\delta}{\delta h} \Gamma_{L}, \quad v_{L}(h, b)=-30 \pi \frac{\delta}{\delta b} \Gamma_{L},
$$

where $\Gamma_{L}[h, b]$ is a reference functional which, up to a change of variables, we obtained in closed, explicit form in [5]. It starts out with

$$
\Gamma_{L}[h, b]=\frac{-1}{2 \pi} \int d^{2} z\left(h \frac{\partial_{-}^{3}}{\partial_{+}} h+\frac{1}{30} b \frac{\partial_{-}^{5}}{\partial_{+}} b+\ldots\right) .
$$


A related functional $W_{L}[u, v]$ is obtained from $\Gamma_{L}[h, b]$ by a Legendre transformation

$$
W_{L}[u, v]=\Gamma_{L}[h(u, v), b(u, v)]+\frac{1}{\pi} \int\left(h u+\frac{1}{30} b v\right),
$$

where $h(u, v)$ and $b(u, v)$, which we denote by $h_{L}(u, v)$ and $b_{L}(u, v)$ for later reference, are determined through the relations in (7.5) and we have that

$$
h_{L}(u, v)=\pi \frac{\delta}{\delta u} W_{L}, \quad b_{L}(u, v)=30 \pi \frac{\delta}{\delta v} W_{L} .
$$

Let us now consider the original problem and consider the induced action for finite c. We decompose

$$
\begin{aligned}
\Gamma_{\text {ind }}^{W_{3}}[h, b]=\frac{c}{12} \Gamma_{L}[h, b] & +\left(b^{4} \text { terms, plus } b^{4} h, b^{4} h^{2}, \ldots, b^{6}, b^{6} h, \ldots \text { terms }\right) \\
& +\frac{1}{c} \text { terms }+\frac{1}{c^{2}} \text { terms }+\ldots
\end{aligned}
$$

and expand $u(h, b)$ and $v(h, b)$ (as defined in (5.12)) as

$$
\begin{aligned}
& u(h, b)=u_{L}(h, b)+\frac{1}{c}\left(b^{4} \text { terms }+b^{4} h, \ldots, b^{6}, \ldots\right)+\frac{1}{c^{2}}\left(b^{6}, \ldots\right) \\
& v(h, b)=v_{L}(h, b)+\frac{1}{c}\left(b^{3} \text { terms }+b^{3} h, \ldots, b^{5}, \ldots\right)+\frac{1}{c^{2}}\left(b^{5}, \ldots\right) .
\end{aligned}
$$

(The $b^{2}$ terms in $u$ are accounted for by $u_{L}$.)

The full result for the $b^{4}$ terms in the induced action was computed in [4], where we directly computed the four-point correlator $\langle W W W W\rangle$. The result is

$$
\Gamma_{\text {ind }}\left[h=0, b^{4}\right]=-\frac{c}{60 \cdot 6 !} \frac{1}{\pi}[I]-\frac{2 \beta c}{5 \cdot 7 !} \frac{1}{\pi}[I I],
$$

where the two structures $[I]$ and $[I I]$ are given by

$$
\begin{aligned}
& {[I]=\int\left(2 b \frac{\partial_{-}^{3}}{\partial_{+}} b-3 \partial_{-} b \frac{\partial_{-}^{2}}{\partial_{+}} b+3 \partial_{-}^{2} b \frac{\partial_{-}}{\partial_{+}} b-2 \partial_{-}^{3} b \frac{1}{\partial_{+}} b\right) \frac{1}{\partial_{+}}\left(2 b \frac{\partial_{-}^{6}}{\partial_{+}} b+3 \partial_{-} b \frac{\partial_{-}^{5}}{\partial_{+}} b\right)} \\
& {[I I]=\int\left(b \frac{\partial_{-}}{\partial_{+}} b-\partial_{-} b \frac{1}{\partial_{+}} b\right) \frac{1}{\partial_{+}}\left(b \frac{\partial_{-}^{8}}{\partial_{+}} b+6 \partial_{-} b \frac{\partial_{-}^{7}}{\partial_{+}} b+14 \partial_{-}^{2} b \frac{\partial_{-}^{6}}{\partial_{+}} b+14 \partial_{-}^{3} b \frac{\partial_{-}^{5}}{\partial_{+}} b\right) .}
\end{aligned}
$$


We remark that the last term in (7.11) corresponds to the lowest nonlocal contribution (which is of order $b^{3}$ ) to the $\lambda$ Ward identity (see (7.1)-(7.3)). To prove this, one may use that taking $\frac{\delta}{\delta b}$ of the expression $[I I]$ is equivalent to 4 times varying w.r.t. the $b$ at the second position.

To lowest order in $1 / c$, the last term of (7.11) is $-\frac{32}{25.71} \frac{1}{\pi}[I I]$. In the following sections we will explicitly show that this term will get cancelled when we pass from the induced to the effective action.

\section{The effective action.}

We now turn to the effective action, and will argue that its Ward identities are local in terms of $h, b, u$ and $v$. The effective action is obtained as follows. First consider the path-integral

$$
\exp W_{\text {conn }}^{\dot{W}_{3}}[t, w]=\int D h D b \exp \left(\Gamma_{\text {ind }}^{W_{3}}[h, b]+\frac{1}{\pi} \int(h t+b w) d^{2} z\right)
$$

It represents the connected Feynman diagrams with propagating $h$ and $b$ fields. Then take the Legendre transform

$$
\Gamma_{\mathrm{eff}}^{W_{3}}[h, b]=W_{\mathrm{conn}}^{W_{3}}[t, w]-\frac{1}{\pi} \int(h t+b w) d^{2} z
$$

where $t(h, b)$ and $w(h, b)$ are the solutions of

$$
\pi \frac{\delta W_{\mathrm{conn}}^{W_{3}}}{\delta t}=h, \quad \pi \frac{\delta W_{\mathrm{conn}}^{W_{3}}}{\delta w}=b
$$

Then $\Gamma_{\text {eff }}^{W_{3}}$ corresponds to all graphs which cannot be split into two parts by cutting one internal $h$ or $b$ line. We shall from now on deal with $W_{\text {conn }}^{W_{3}}[t, w]$. If one wishes $\Gamma_{\mathrm{eff}}^{W_{3}}$ instead, one must make the above Legendre transformation.

We evaluate $W_{\text {conn }}^{W_{3}}[t, w]$ in an $h, b$-loop expansion as follows. First we take the saddle point approximation. The saddle point are those functions $h_{0}(t, w)$ and $b_{o}(t, w)$ where the integrand is stationary

$$
\left.\begin{array}{l}
\frac{\delta}{\delta h} \Gamma_{\text {ind }}^{W_{3}}[h, b]+\frac{1}{\pi} t=0 \\
\frac{\dot{\delta}}{\delta b} \Gamma_{\text {ind }}^{W_{3}}[h, b]+\frac{1}{\pi} w=0
\end{array}\right\} \text { solution }: \begin{aligned}
& h=h_{0}(t, w) \\
& b=b_{0}(t, w)
\end{aligned}
$$


Comparing with (5.12), (7.5) and (7.9), we find

$$
\begin{aligned}
& h_{o}(t, w)=h_{L}\left(u=\frac{12}{c} t, v=\frac{360}{c} w\right)+\mathcal{O}\left(\frac{1}{c}\right), \\
& b_{o}(t, w)=b_{L}\left(u=\frac{12}{c} t, v=\frac{360}{c} w\right)+\mathcal{O}\left(\frac{1}{c}\right) .
\end{aligned}
$$

Then we decompose $h$ and $b$ as follows

$$
h=h_{0}(t, w)+h^{\prime}, \quad b=b_{0}(t, w)+b^{\prime}
$$

and expand the exponent in terms bilinear in the quantum fields $h^{\prime}, b^{\prime}$, terms trilinear in $h^{\prime}, b^{\prime}$, etc. Hence,

$$
e^{W_{\text {conn }}^{W_{3}}[t, w]}=e^{W_{\text {saddle }}^{W_{3}}[t, w]} \int D h^{\prime} D b^{\prime} e^{I^{(2)}}+I^{(3)}+\ldots,
$$

where

$$
\begin{aligned}
W_{\text {saddle }}^{W_{3}}[t, w] & =\Gamma_{\text {ind }}^{W_{3}}\left[h_{0}, b_{0}\right]+\frac{1}{\pi} \int\left(h_{0} t+b_{0} w\right) d^{2} z, \\
I^{(2)} & =\frac{1}{2 !} \int\left(h^{\prime} b^{\prime}\right)\left(\begin{array}{cc}
\frac{\delta u}{\delta h} & \frac{\delta u}{\delta b} \\
\frac{\delta v}{\delta h} & \frac{\delta v}{\delta b}
\end{array}\right)\left(\begin{array}{c}
h^{\prime} \\
b^{\prime}
\end{array}\right) d^{2} z
\end{aligned}
$$

We have dropped the factors $\frac{c}{12}$ and $\frac{c}{360}$ to the power infinity (see (5.12)), as they are additive constants in $W_{\text {conn }}^{W_{3}}$. Clearly, the saddle point approximation of $W_{\text {conn }}^{W_{3}}$ is the Legendre transform of $\Gamma_{\text {ind }}^{W_{3}}$.

The leading order terms in $W_{\text {conn }}^{W_{3}}[t, w]$ are known; they are given by the Legendre transform of $\frac{c}{12} \Gamma_{L}[h, b]$. We are interested in the order $1 / c$ corrections to $W_{\text {conn }}^{W_{3}}[t, w]$ since we have seen that similar terms lead to non-localities in the Ward identity (7.1) for the induced action. $W_{\text {conn }}^{W_{3}}[t, w]$ depends on $t$ and $w$ through the explicit $t$ and $w$ appearing in $\left(h_{0} t+b_{0} w\right)$ and through $h_{0}(t, w)$ and $b_{0}(t, w)$. [A little thinking shows that, for the purpose of considering $1 / c$ corrections to the saddle-point result, we may replace $h_{o}(t, w)$ by $h_{L}\left(u=\frac{12}{c} t, v=\frac{360}{c} w\right)$ and $b_{o}(t, w)$ by $b_{L}\left(u=\frac{12}{c} t, v=\frac{360}{c} w\right.$ ) (compare with (8.5). Coriversely, one may replace the saddle-point value for $t$ and $w$, to be denoted by $t_{0}(h, b)$ and $w_{0}(h, b)$ by $\frac{c}{12} u_{L}(h, b)$ and $\frac{c}{360} v_{L}(h, b)$. We will use this in sections 11, 12.] 
Since the kinetic terms for $h$ and $b$ are proportional to $c$, whereas the interactions are proportional to $c$ or down by powers of $1 / c$, we can interpret $1 / c$ as the Planck's constant. The action $\Gamma_{\text {ind }}^{W_{3}}$ is thus: a 'classical action' $\left(\frac{c}{12} \Gamma_{L}\right)$ plus ' $\hbar$ corrections' (the term with $c^{0}$ ) plus $\hbar^{2}$ corrections (term with $1 / c$ ), etc. It follows that the leading 1-loop results give order $1 / c$ corrections to the saddle-point result.

In what follows we will focus on the $c^{0} b_{0}^{4}$ terms in $W_{\text {conn }}^{W_{3}}$. They come from two sources

(i) from the $b^{4}$ term in $\Gamma_{\text {ind }}^{W_{3}}$ in (7.11), with $b$ replaced by $b_{0}$,

(ii) from the 1-loop corrections to the path-integral.

\section{1-Loop corrections to $W_{\text {conn }}^{W_{3}}$.}

The complete 1-loop correction to the saddle-point approximation is given by

$$
K=-\frac{1}{2} \ln \operatorname{det}\left(\begin{array}{cc}
\frac{\delta u}{\delta h} & \frac{\delta v}{\delta h} \\
\frac{\delta u}{\delta b} & \frac{\delta v}{\delta b}
\end{array}\right) \quad \text { at } h=h_{0}, b=b_{0} .
$$

In here we may replace $u, v$ by $u_{L}$ and $v_{L}$ since we only want the $c^{0}$ terms (i.e., we keep only the vertices in $\Gamma_{\text {ind }}^{W_{3}}$ which are of order $c$ ). Since $u_{L}$ and $v_{L}$ satisfy the Ward identities (7.4) we can find an equation for the entries $\frac{\delta u_{h}}{\delta h}$ etc., by differentiating each of these Ward identities w.r.t. $h$ or $b$. One finds then an equation of the form

$$
M\left(\begin{array}{cc}
\frac{\delta u_{h}}{\delta h} & \frac{\delta u_{h}}{\delta b} \\
\frac{\delta v_{h}}{\delta h} & \frac{\delta v_{h}}{\delta b}
\end{array}\right)=N
$$

and hence

$$
K=\frac{1}{2} \ln \operatorname{det}\left(N^{-1} M\right)=\frac{1}{2} \ln \operatorname{det} M-\frac{1}{2} \ln \operatorname{det} N .
$$

The explicit form of the operator-valued matrices $M$ and $N$ is

$$
M=\left[\begin{array}{cc}
\nabla_{+}^{(2)} & -\left(\frac{1}{10} b^{\prime}+\frac{1}{15} b \partial_{-}\right) \\
-L & \nabla_{+}^{(3)}
\end{array}\right]\left[\begin{array}{cc}
\delta^{2}(z-w) & 0 \\
0 & \delta^{2}(z-w)
\end{array}\right]
$$




$$
N=\left[\begin{array}{cc}
D_{1} & \left(\frac{1}{10} v \partial_{--}+\frac{1}{15} v^{\prime}\right) \\
3 v \partial_{-}+v^{\prime} & D_{2}
\end{array}\right]\left[\begin{array}{cc}
\delta^{2}(z-w) & 0 \\
0 & \delta^{2}(z-w)
\end{array}\right]
$$

where

$$
\nabla_{+}^{(j)}=\partial_{+}-h \partial_{-}-j h^{\prime}
$$

and

$$
L=\left(10 b^{\prime \prime \prime}+15 b^{\prime \prime} \partial_{-}+9 b^{\prime} \partial_{-}^{2}+2 b \partial_{-}^{3}\right)+\left(32 u b^{\prime}+16 b u^{\prime}+16 b u \partial_{-}\right) .
$$

Our task is to compute each of these two determinants. This is similar to the evaluation of the Jacobians for anomalies, and we must similarly regulate these expressions. We will use the well-known representation of determinants as Gaussian integrals of anticommuting variables and write the determinants as the partition functions of certain 'b-c' systems. ${ }^{10}$ We introduce anticommuting fields $b_{1}, b_{2}, c_{1}, c_{2}$ for $M$, and $B_{1}, B_{2}, C_{1}, C_{2}$ for $N$, and write

$$
\operatorname{det} M=\int d c_{1} d b_{1} d c_{2} d b_{2} e^{\frac{1}{\pi} \int\left(b_{1} b_{2}\right) M\left(\begin{array}{l}
c_{1} \\
c_{2}
\end{array}\right) d^{2} z}
$$

and a similar expression for $N$.

We now write the exponent (the 'action') in this expression as

$$
I^{(M)}=\frac{1}{\pi} \int\left(b_{1} b_{2}\right) M\left(\begin{array}{l}
c_{1} \\
c_{2}
\end{array}\right) d^{2} z=I_{\text {free }}^{(M)}+I_{\text {int }}^{(M)},
$$

where

$$
\begin{aligned}
I_{\text {free }}^{(M)} & =\frac{1}{\pi} \int\left(b_{1} \partial_{+} c_{1}+b_{2} \partial_{+} c_{2}\right) d^{2} z \\
I_{\text {int }}{ }^{(M)} & =-\frac{1}{\pi} \int\left(h T_{h}+b T_{b}+b u T_{b u}+b^{\prime} u T_{b^{\prime} u}\right) d^{2} z
\end{aligned}
$$

and

$$
T_{h}=-b_{1} c_{1}^{\prime}-2 b_{1}^{\prime} c_{1}-2 b_{2} c_{2}^{\prime}-3 b_{2}^{\prime} c_{2}
$$

\footnotetext{
${ }^{10}$ Equivalently, we could have taken a commuting $b-c$ system to obtain the inverses of the determinants.
} 


$$
\begin{aligned}
T_{b} & =-\frac{1}{30}\left(b_{1} c_{2}^{\prime}+3 b_{1}^{\prime} c_{2}\right)+\left(10 b_{2}^{\prime \prime \prime} c_{1}+15 b_{2}^{\prime \prime} c_{1}^{\prime}+9 b_{2}^{\prime} c_{1}^{\prime \prime}+2 b_{2} c_{1}^{\prime \prime \prime}\right) \\
T_{b u} & =-16 b_{2}^{\prime} c_{1} \\
T_{b^{\prime} u} & =16 b_{2} c_{1} .
\end{aligned}
$$

Hence

$$
\operatorname{det} M=\left\langle e^{-\frac{1}{\pi} \int d^{2} z\left(h T_{h}+b T_{b}+b u T_{b u}+b^{\prime} u T_{b^{\prime} u}\right)}\right\rangle .
$$

The action for $N$ analogous to (9.8) is given by

$$
I^{(N)}=\frac{1}{\pi} \int\left(B_{1} B_{2}\right) N\left(\begin{array}{l}
C_{1} \\
C_{2}
\end{array}\right) d^{2} z .
$$

It can be written as $I=I_{\text {free }}+I_{\text {int }}$, where

$$
\begin{aligned}
I_{\text {free }}^{(N)} & =\frac{1}{\pi} \int\left(B_{1} \partial_{-}^{3} C_{1}+B_{2} \partial_{-}^{5} C_{2}\right) d^{2} z \\
I_{\text {int }}^{(N)} & =\frac{1}{\pi} \int\left(u H_{u}+v H_{v}+u^{2} H_{u u}\right) d^{2} z
\end{aligned}
$$

and

$$
\begin{aligned}
H_{u}= & \left(B_{1} C_{1}^{\prime}-B_{1}^{\prime} C_{1}\right)+10 B_{2} C_{2}^{\prime \prime \prime}-15 \partial_{-}\left(B_{2} C_{2}^{\prime \prime}\right) \\
& +9 \partial_{-}^{2}\left(B_{2} C_{2}^{\prime}\right)-2 \partial_{-}^{3}\left(B_{2} C_{2}\right) \\
= & \left(B_{1} \overleftrightarrow{\partial_{-}} C_{1}\right)+\left(2 B_{2} C_{2}^{\prime \prime \prime}-3 B_{2}^{\prime} C_{2}^{\prime \prime}+3 B_{2}^{\prime \prime} C_{2}^{\prime}-2 B_{2}^{\prime \prime \prime} C_{2}\right) \\
H_{v}= & \frac{1}{30}\left(B_{1} C_{2}^{\prime}-2 B_{1}^{\prime} C_{2}\right)+\left(2 B_{2} C_{1}^{\prime}-B_{2}^{\prime} C_{1}\right) \\
H_{u u}= & 8\left(B_{2} C_{2}^{\prime}-B_{2}^{\prime} C_{2}\right) .
\end{aligned}
$$

Hence the expression for $W_{\text {conn }}^{W_{3}}[t, w]$ through 1-loop reads

$$
\begin{aligned}
e^{W_{\text {conn }}^{W_{3}}[t, w]}= & e^{W_{\text {saddle }}^{W_{3}}[t, w]}\left(\left\langle e^{-\frac{1}{\pi} \int\left(h T_{h}+b T_{b}+b u T_{b u}+b^{\prime} u T_{b^{\prime} u}\right) d^{2} z}\right\rangle\right)^{1 / 2} \\
& \times\left(\left\langle e^{\frac{1}{\pi} \int\left(u H_{u}+v H_{v}+u^{2} H_{u u}\right) d^{2} z}\right\rangle\right)^{-1 / 2}
\end{aligned}
$$


It would be nice if we could write down a pair of Ward identities for the partition functions $\ln \operatorname{det} M$, and $\ln \operatorname{det} N$ and then solve them exactly. For the $c \rightarrow \pm \infty$ limit of the Ward identities for $\Gamma_{\text {ind }}^{W_{3}}$ we were able to achieve this, because in this limit the Ward identities became local (albeit nonlinear) differential equations (anyhow, it was a hard job). For the present case, it seems hopeless to follow this path, as the Ward identities are expected to be nonlocal (and nonlinear): their nonlocalities are, after all, supposed to cancel the original nonlocalities in $\Gamma_{\text {ind }}^{W_{3}}$.

To get an idea how to proceed to obtain $\ln \operatorname{det} M$ and $\ln \operatorname{det} N$, we now first consider the truncation to pure gravity. In this case,

$$
\begin{aligned}
M^{\text {grav }}=\nabla_{+}^{(2)} \delta^{2}(z-w), & \nabla_{+}^{(2)}=\partial_{+}-h \partial_{-}-2 h^{\prime}, \\
N^{\text {grav }}=D_{1} \delta^{2}(z-w), & D_{1}=\partial_{-}^{3}+2 u \partial_{-}+u^{\prime} .
\end{aligned}
$$

The results for $\operatorname{det} M^{\text {grav }}$ and $\operatorname{det} N^{\text {grav }}$ will then suggest what the leading terms in the result for $\operatorname{det} M$ and $\operatorname{det} N$ will be.

\section{The determinants in pure gravity.}

Let us first consider the most complicated case, which is the truncation of $N$ to pure gravity: $N^{\text {grav }}=D_{1} \delta^{2}(z-w)$. We have

$$
I_{\text {free }}^{(N)}=-\frac{1}{\pi} \int B \partial_{-}^{3} C, \quad I_{\text {int }}^{(N)}=-\frac{1}{\pi} \int u J, \quad J=B C^{\prime}-B^{\prime} C .
$$

We claim that the propagators are given by ${ }^{11}$

$$
\langle C(z, \bar{z}) B(w, \bar{w})\rangle=\langle B(z, \bar{z}) C(w, \bar{w})\rangle=-\frac{1}{2} \frac{(z-w)^{2}}{\bar{z}-\bar{w}} .
$$

Assuming for a moment this to be the case, we obtain the OPE

$$
J(z, \bar{z}) J(w, \bar{w})=-\frac{(z-w)^{2}}{(\bar{z}-\bar{w})^{2}}-\frac{(z-w)}{\bar{z}-\bar{w}} J(w, \bar{w})-\frac{1}{2} \frac{(z-w)^{2}}{\bar{z}-\bar{w}} J^{\prime}(w, \bar{w}) .
$$

\footnotetext{
${ }^{11}$ Since $B$ and $C$ have total conformal dimension -1 and total conformal spin +3 , the propagator must be of the form $(z-w)^{2} /(\bar{z}-\bar{w})$.
} 
The truncation of this OPE at the position shown here should be compared to the 'contraction' or 'singular OPE' and is such that Wick-decomposition of correlation functions holds. The truncation of course corresponds to the on-shell condition $\partial_{-}^{3} J=0$.

Let us now come back to the propagators (10.2). In general, propagators are obtained by adding the source terms $\frac{1}{\pi} \int\left(j_{C} C+j_{B} B\right) d^{2} z$ to the free action and completing squares. This yields $K=-\frac{1}{\pi} \int j_{C} \frac{1}{\partial_{-}^{3}} j_{B} d^{2} z$. Then $\langle C(z, \bar{z}) B(w, \bar{w})\rangle=-\pi^{2} \frac{\delta}{\delta j_{C}(z)} \frac{\delta}{\delta j_{B}(w)}$ $\exp K=-\pi \frac{1}{\partial^{3}} \delta^{2}(z-w)$. Comparing with (10.2), we should thus prove the distribution identity $^{12}$

$$
\pi \frac{1}{\partial_{-}^{3}} \delta^{2}(z-w)=\lim _{\epsilon 10} \frac{1}{2} \frac{(z-w)^{2}}{(\bar{z}-\bar{w})} \theta\left[(z-w)(\bar{z}-\bar{w})-\epsilon^{2}\right] .
$$

To prove this, we multiply by a test function $\partial_{-}^{3} f(w, \bar{w})$ and integrate over $d^{2} w$. On the l.h.s. this gives $\pi f(z, \bar{z})$, while on the r.h.s. we find

$$
\text { r.h.s. }=\lim _{e \downarrow 0} \int d^{2} w \frac{1}{2} f(w, \bar{w}) \partial_{w}^{3}\left\{-\frac{(z-w)^{2}}{\bar{z}-\bar{w}} \theta\left[(z-w)(\bar{z}-\bar{w})-\epsilon^{2}\right]\right\}
$$

Since $(z-w)(\bar{z}-\bar{w})=\rho^{2}$ is larger than $\epsilon^{2}$, the $\partial_{w}$ derivatives annihilate $(\bar{z}-\bar{w})^{-1}$, and we get terms with 1,2 or $3 \partial_{w}$ derivatives on the $\theta$ function. They yield, respectively,

$$
\begin{aligned}
6 & \left(\frac{1}{\bar{z}-\bar{w}}\right)(\bar{z}-\bar{w}) \delta\left(\rho^{2}-\epsilon^{2}\right)-6 \frac{z-w}{\bar{z}-\bar{w}} \partial_{w}\left\{(\bar{z}-\bar{w}) \delta\left(\rho^{2}-\epsilon^{2}\right)\right\} \\
& +\frac{(z-w)^{2}}{(\bar{z}-\bar{w})} \partial_{w}^{2}\left\{(\bar{z}-\bar{w}) \delta\left(\rho^{2}-\epsilon^{2}\right)\right\} \\
= & 6 \delta\left(\rho^{2}-\epsilon^{2}\right)-6(z-w) \partial_{w} \delta\left(\rho^{2}-\epsilon^{2}\right)+(z-w)^{2} \partial_{w}^{2} \delta\left(\rho^{2}-\epsilon^{2}\right) .
\end{aligned}
$$

Putting this into (10.5), and integrating by parts, it yields indeed $\int d^{2} w \frac{1}{2} f(w, \bar{w}) 2 \delta\left(\rho^{2}-\right.$ $\left.\epsilon^{2}\right)=\pi f(z, \bar{z})$.

We derive, as before, a Ward identity for $\operatorname{det} N^{\text {grav }}=\left\langle\exp \frac{1}{\pi} \int u J d^{2} z\right\rangle \equiv \exp \Gamma_{\text {ind }}^{(N)}[u]$. We recall that $u=\frac{\partial^{3}}{\partial_{+}} h+\ldots$ and begin by varying $\delta u=\partial_{-}^{3} \epsilon$. This yields

$$
\left(\delta \Gamma_{\text {ind }}^{(N)}\right) \exp \Gamma_{\text {ind }}^{(N)}=\left\langle\left(\frac{1}{\pi} \int \partial_{-}^{3} \epsilon J d^{2} z_{1}\right)\left(\frac{1}{\pi} \int u J d^{2} z_{2}\right) e^{\frac{1}{\pi} \int u J d^{2} z}\right\rangle .
$$

\footnotetext{
${ }^{12}$ Note that one recovers (5.6) for $n=1$ by acting with $\partial_{-}^{2}$.
} 
Using the contraction for $J J$ given above and distribution identities similar to (10.4) we obtain

$$
\left(\delta \Gamma_{\text {ird }}^{(N)}\right) \exp \Gamma_{\text {ind }}^{(N)}=\left\langle\left[\frac{1}{\pi} \int\left(-u \epsilon^{\prime}+\epsilon u \partial_{-}\right) J+\frac{2}{\pi}\left(\partial_{+} \epsilon\right) u\right] d^{2} z_{2} e^{\frac{1}{\pi} \int u J d^{2} z}\right\rangle .
$$

By adding a suitable term to $\delta u$, we remove the terms with $J$, after which only the minimal anomaly (i.e., with one $u$ ) remains. Discarding the local parameter $\epsilon$, we arrive at the following Ward identity

$$
\left(\partial_{-}^{3}+2 u \partial_{-}+u^{\prime}\right) \frac{\delta}{\delta u} \Gamma_{\text {ind }}^{(N)}=\frac{2}{\pi} \partial_{+} u .
$$

Do we recognize this identity? The induced action of pure gravity, defined by

$$
e^{\Gamma_{\mathrm{ind}}^{\mathrm{grav}}[h]}=\left\langle e^{-\frac{1}{\pi} \int h T d^{2} z}\right\rangle
$$

satisfies

$$
\left(\partial_{+}-h \partial_{-}-2 h^{\prime}\right) \frac{\delta}{\delta h} \Gamma_{\mathrm{ind}}^{\mathrm{grav}}=-\left(\frac{c}{12 \pi}\right) \partial_{-}^{3} h .
$$

Let us turn this relation 'inside out' by using the Legendre transform,

$$
\begin{gathered}
t=-\pi \frac{\delta}{\delta h} \Gamma_{\text {ind }}^{\text {grav }}, \quad h=\pi \frac{\delta}{\delta t} W_{\text {ind }}^{\text {grav }} \\
W_{\text {ind }}^{\text {grav }}[t]=\Gamma_{\text {ind }}^{\text {grav }}[h(t)]+\frac{1}{\pi} \int h t d^{2} z,
\end{gathered}
$$

To make contact with (10.9), we consider suitably normalized functionals as in (7.7)

$$
\Gamma_{L}^{\text {grav }}[h]=\frac{12}{c} \Gamma_{\text {ind }}^{\text {grav }}[h], \quad W_{L}^{\text {grav }}[u]=\frac{12}{c} W_{\text {ind }}^{\text {grav }}\left[t=\frac{c}{12} u\right]
$$

with

$$
W_{L}^{\text {grav }}[u]=\Gamma_{L}^{\text {grav }}[h]+\frac{1}{\pi} \int h u d^{2} z
$$


Then (10.11) becomes

$$
\frac{1}{\pi} \partial_{+} u-\left[u^{\prime}+2 u \partial_{-}\right] \frac{\delta}{\delta u} W_{L}^{\text {grav }}=\partial_{-}^{3}\left[\frac{\delta}{\delta u} W_{L}^{\text {grav }}\right] .
$$

Comparison of (10.15) and (10.11) reveals that

$$
\ln \operatorname{det} N=\Gamma_{\text {ind }}^{(N)}[u]=2 W_{L}^{\text {grav }}[u]
$$

Thus we see that, up to a constant, $\ln \operatorname{det} N$ is given by the Legendre transform of the induced action of pure gravity.

Next we consider the truncation of $M$ to pure gravity. We write

$$
\operatorname{det}\left(\partial_{+}-2 h^{\prime}-h \partial_{-}\right)=\int d c d b e^{\frac{1}{\pi}} \int\left(b \partial_{+} c-h T_{h}\right) d^{2} z
$$

where

$$
T_{h}=-b c^{\prime}-2 b^{\prime} c
$$

The propagators are $\langle c(z) b(w)\rangle=-\frac{1}{z-w}$ and $T_{h}$ is just the stress tensor for coordinate ghosts

$$
T_{h}(z) T_{h}(w)=\frac{-13}{(z-w)^{4}}+\frac{2 T_{h}(w)}{(z-w)^{2}}+\frac{T_{h}^{\prime}(w)}{z-w} .
$$

The Ward identity for

$$
\operatorname{det} M^{\mathrm{grav}}=e^{\Gamma_{\mathrm{ind}}^{(M)}}=\left\langle e^{-\frac{1}{\pi} \int h T_{h} d^{2} z}\right\rangle
$$

follows from varying $\delta h=\partial_{+} \epsilon$ and then using $T_{h} T_{h}$ to obtain extra $\delta h$ terms which cancel everything except the minimal anomaly. One finds with (5.6)

$$
\left(\partial_{+}-h \partial_{-}-2 h^{\prime}\right) \frac{\delta}{\delta h} \Gamma_{\mathrm{ind}}^{(M)}=\frac{26}{12 \pi} \partial_{-}^{3} h
$$


Comparison with (10.11) immediately yields

$$
\ln \operatorname{det} M=\Gamma_{\mathrm{ind}}^{(M)}[h]=-\frac{13}{6} \Gamma_{L}^{\mathrm{grav}}[h] .
$$

Hence, $\operatorname{det} M$ is proportional to the induced action itself.

For later reference we mention the results for the determinants of the operators $\nabla_{+}^{(j)}$ and $D_{j}$, which are the spin-j analogs of the operators $M=\nabla_{+}^{(2)}$ and $N=D_{1}$ discussed here. We have $[31,37]$

$$
\begin{aligned}
\ln \operatorname{det} \nabla_{+}^{(j)} & =-\frac{\left(6 j^{2}-6 j+1\right)}{6} \Gamma_{L}^{\mathrm{grav}}[h] \\
\ln \operatorname{det} D_{j} & =\frac{j(2 j+1)(2 j+2)}{6} W_{L}^{\mathrm{grav}}[u] .
\end{aligned}
$$

\section{The determinants in $W_{3}$ gravity.}

Based on the results in the previous section, we can easily find some first results for the $W_{3} \operatorname{determinants} \operatorname{det} M$ and $\operatorname{det} N$. If we put $b=0$ in $\operatorname{det} M$ and $v=0$ in $\operatorname{det} N$, the matrices $M$ and $N$ reduce to diagonal matrices, whose determinant we easily find [37]:

$$
\begin{aligned}
& \ln \operatorname{det} M[h, b=0]=\ln \operatorname{det} \nabla_{+}^{(2)}+\ln \operatorname{det} \nabla_{+}^{(3)}=\frac{(-13-37)}{6} \Gamma_{L}^{\text {grav }}[h] \\
& \ln \operatorname{det} N[u, v=0]=\ln \operatorname{det} D_{1}+\ln \operatorname{det} D_{2}=(2+10) W_{L}^{\text {grav }}[u] .
\end{aligned}
$$

One may expect that putting in the $b$ and $v$ dependences will simply extend these results to $\ln \operatorname{det} M=-\frac{50}{6} \Gamma_{L}[h, b]$ and $\ln \operatorname{det} N=12 W_{L}[u, v]$. However, we will find below that things are not as simple as that. Let us now work out these determinants in more detail.

We first focus on $\ln \operatorname{det} M$. Up to a change in notation, the currents $T_{h}$ and $T_{b}$ in (9.10) are very similar to the the currents $T^{\text {gh }}$ and $W^{\text {gh }}$ that one finds in a BRST treatment of critical $(c=100) W_{3}$ gravity (see section 4$)$. However, in this case the current $T_{b}$ is not a primary current with respect to $T_{h}$, and the $T_{b}-T_{b}$ OPE is modified accordingly. One finds

$$
T_{h}(z) T_{h}(w)=\frac{-50}{(z-w)^{4}}+\frac{2 T_{h}(w)}{(z-w)^{2}}+\frac{\partial_{-} T_{h}(w)}{(z-w)}+\ldots
$$




$$
\begin{aligned}
T_{h}(z) T_{b}(w)= & \frac{388 b_{2} c_{1}(w)}{(z-w)^{5}}+\frac{96\left(b_{2} \partial_{-} c_{1}+2 \partial_{-} b_{2} c_{1}\right)(w)}{(z-w)^{4}}+\frac{3 T_{b}(w)}{(z-w)^{2}}+\frac{\partial_{-} T_{b}(w)}{(z-w)}+\ldots \\
T_{b}(z) T_{b}(w)= & -\frac{348}{5} \frac{1}{(z-w)^{6}}+\frac{2 T_{h}(w)}{(z-w)^{4}}+\frac{\partial_{-} T_{h}(w)}{(z-w)^{3}} \\
& +\frac{1}{(z-w)^{2}}\left[\frac{3}{10} \partial_{-}^{2} T_{h}(w)-\frac{16}{15} \partial_{-}^{3} b_{1} c_{1}(w)\right] \\
& +\frac{1}{(z-w)}\left[\frac{1}{15} \partial_{-}^{3} T_{h}(w)-\frac{8}{15} \partial_{-}\left(\partial_{-}^{3} b_{1} c_{1}\right)(w)\right]+\ldots
\end{aligned}
$$

The $T_{h}-T_{h}$ OPE shows that the functional $-\frac{50}{6} \Gamma_{L}[h, b]$ indeed reproduces the $b$ independent terms in the logarithm of the determinant. However, since the current algebra (11.2) is significantly different from the exact $W_{3}$ algebra at $c=-100$, we can not expect that $-\frac{50}{6} \Gamma_{L}[h, b]$ is the exact result to all orders in $b$. For example, in the exact $c=-100 W_{3}$ algebra, the coefficient of the pole $(z-w)^{-6}$ in the OPE $T_{b}-T_{b}$ is $-100 / 3$, rather than the value $-348 / 5$ that we find here. This implies that already at the level of the terms quadratic in $b$, the logarithm of $\operatorname{det} M$ deviates from $-\frac{50}{6} \Gamma_{L}[h, b]$. The extra terms quadratic in $b$ can be written in the following suggestive way

$$
\frac{1}{240}\left(\frac{348}{5}-\frac{100}{3}\right) \frac{1}{\pi} \int d^{2} z b \frac{\partial_{-}^{5}}{\partial_{+}} b=\frac{272}{5 \pi c} \int d^{2} z w_{0}(h, b) b+\ldots
$$

where we used (see section 8) that $w_{0}(h, b)=\frac{c}{360} v_{L}(h, b)+\ldots=\frac{c}{360} \frac{\partial_{-}^{5}}{\partial_{+}} b+\ldots$ at the saddlepoint. We will later see that also certain higher orders in $b$ can be reproduced by the right hand side of (11.3).

If we now look at the terms of order $b^{2} h$, we find contributions from (i) the 3-point function $\left\langle T_{b} T_{b} T_{h}\right\rangle$ and (ii) the 2-point functions $\left\langle T_{b} T_{b^{\prime} u}\right\rangle$ and $\left\langle T_{b} T_{b u}\right\rangle$, where in the latter two cases we use that $u$ is given by $u_{L}(h, b)=\frac{\partial_{-}^{3}}{\partial_{+}} h+\ldots$. It so turns out that the terms (ii) precisely cancel the terms in (i) that come from the anomalous part of the OPE $T_{h}-T_{b}$. The remaining terms are precisely described by $-\frac{50}{6} \Gamma_{L}[h, b]$ plus the $b^{2} h$ terms in the correction term (11.3).

In a similar way one can compute, order by order, higher terms in the logarithm of $\operatorname{det} M$. As announced in section 8 , we now discuss the terms of order $b^{4}$. These arise from (i) the 4-point function $\left\langle T_{b} T_{b} T_{b} T_{b}\right\rangle$ and (ii) the 2-point functions $\left\langle T_{b} T_{b^{\prime} u}\right\rangle$ and $\left.<T_{b} T_{b u}\right\rangle$, where in the latter we now use the part of the saddle-point value $u_{L}(h, b)$ 
that is quadratic in $b[4]$ :

$$
u(h, b)=\ldots+\frac{1}{30} \frac{1}{\partial_{+}}\left(3 \partial_{-} b \frac{\partial_{-}^{5}}{\partial_{+}} b+2 b \frac{\partial_{-}^{6}}{\partial_{+}} b\right)+\ldots
$$

After a straightforward though rather lengthy computation one finds that all the $b^{4}$ terms can be collected in two terms proportional to the strucutures $[I]$ and $[I I]$ given in (7.12)! One finds

$$
\frac{397}{75 \cdot 6 !} \frac{1}{\pi}[I]+\frac{64}{25 \cdot 7 !} \frac{1}{\pi}[I I] .
$$

The terms proportional to [I] are precisely reproduced by the two terms in $\ln \operatorname{det} M$ which we identified above, leaving us with the terms proportional to structure $[I I]$.

In summary, we find the following result, which is exact through the orders $h^{n}, b^{2}$, $b^{2} h$ and $b^{4}$

$$
\begin{aligned}
& \ln \operatorname{det} M=\ln \operatorname{det}\left(\begin{array}{cc}
\nabla_{+}^{(2)} & -\frac{1}{10}\left(\partial_{-} b\right)-\frac{1}{15} b \partial_{-} \\
-L & \nabla_{+}^{(3)}
\end{array}\right)= \\
& -\frac{50}{6} \Gamma_{L}[h, b]+\frac{272}{5 \pi c} \int d^{2} z w_{0}(h, b) b+\frac{64}{25 \cdot 7 !} \frac{1}{\pi}[I I]+\ldots,
\end{aligned}
$$

where the dots will not affect the abovementioned orders in $h, b$.

For the computation of ln $\operatorname{det} N$, we consider the current algebra of the currents $H_{u}$ and $H_{v}$. We find the following OPE's ${ }^{13}$

$$
\begin{aligned}
H_{u}(z, \bar{z}) H_{u}(w, \bar{w})= & -6 \frac{(z-w)^{2}}{(\bar{z}-\bar{w})^{2}}-\frac{(z-w)}{(\bar{z}-\bar{w})} H_{u}(w, \bar{w})-\frac{1}{2} \frac{(z-w)^{2}}{(\bar{z}-\bar{w})} \partial_{-} H_{u}(w, \bar{w}) \\
& -2 \delta^{2}(z-w) H_{u u}(w, \bar{w})+\ldots \\
H_{u}(z, \bar{z}) H_{v}(w, \bar{w})= & -2 \frac{(z-w)}{(\bar{z}-\bar{w})} H_{v}(w, \bar{w})-\frac{1}{2} \frac{(z-w)^{2}}{(\bar{z}-\bar{w})} \partial_{-} H_{v}(w, \bar{w})+\ldots \\
H_{v}(z, \bar{z}) H_{v}(w, \bar{w})= & \frac{-1}{60} \frac{(z-w)^{4}}{(\bar{z}-\bar{w})^{2}}-\frac{1}{180} \frac{(z-w)^{3}}{(\bar{z}-\bar{w})} H_{u}(w, \bar{w}) \\
& -\frac{1}{360} \frac{(z-w)^{4}}{(\bar{z}-\bar{w})} \partial_{-} H_{u}(w, \bar{w})+\ldots
\end{aligned}
$$

\footnotetext{
${ }^{13}$ To obtain the terms with $\delta^{2}(z-w) H_{u u}(w)$, use that $\left.\partial_{-}^{5}{ }^{\diamond}(z-w)^{4} /(\bar{z}-\bar{w})\right)$ equals $24 \pi \delta^{2}(z-w)$. This comes about because further terms with derivatives of delta functions cancel each other after partial integration.
} 
Note that the singularities in these OPE's are not just functions of the form $\frac{(x-w)^{n}}{(z-w)^{m}}$ (as is the case for pure gravity), but also take the form of bare delta-functions. These lead to terms in the determinant containing a factor $u^{2}$, which combine with similar terms coming from the coupling $u^{2} H_{u u}$ in (9.13). One already encounters this complication when evaluating the determinant of the operator $D_{2}$ defined in (5.21), which is a part of our more complicated operator M. It was suggested in [31] that in the computation of $\ln \operatorname{det} D_{2}$ the different $u^{2}$ terms precisely cancel, so that the final result is simply proporional to $W_{L}^{\text {grav }}[u]$ as in (10.23). We checked this claim for the contributions of the form $\int u^{2} \frac{\partial_{+}}{\partial_{-}^{3}} u$ and $\int u^{2} \frac{\partial_{+}}{\partial_{-}^{2}} u^{2}$ and found it to be correct. In our case we do not expect a complete cancellation of the $u^{2}$ terms, since the Ward identities (7.4) that determine the form of the reference functional $W_{L}[u, v]$ explicitly contain $u^{2}$ terms.

We used the OPE's (11.7) to explicitly compute the leading terms in the logarithm of $\ln \operatorname{det} N$, which are all consistent, up to a factor of 12 , with the WI's (7.4) of the reference functional $W_{L}[u, v]$, so that

$$
\ln \operatorname{det} N=\ln \operatorname{det}\left(\begin{array}{cc}
D_{1} & \frac{1}{10} v \partial_{-}+\frac{1}{15}\left(\partial_{-} v\right) \\
3 v \partial_{-}+\left(\partial_{-} v\right) & D_{2}
\end{array}\right)=12 W_{L}[u, v]+\ldots
$$

Using the saddle-point expressions $u_{L}(h, b)$ and $v_{L}(h, b)$, we can express the result in terms of $h$ and $b$; we checked that (11.8) is exact through the orders $h^{n}, b^{2}, b^{2} h$ and $b^{4}$.

\section{All order result for the effective action.}

We are now ready to combine the results $(7.9),(7.11),(11.6)$ and (11.8) into an expression for the effective action, which is exact through the orders $h^{n}, b^{2}, b^{2} h$ and $b^{4}$ in the leading $1 / c$ correction to the saddle-point result. To our great satisfaction, we find that the explicit non-local structure $[I I]$ precisely cancels between the induced action and the determinant corrections. The remaining terms are

$$
\begin{aligned}
& W_{\mathrm{conn}}^{W_{3}}[t, w]= \\
& \quad \frac{c}{12} W_{L}\left[\frac{12}{c} t, \frac{360}{c} w\right]-6 W_{L}\left[\frac{12}{c} t, \frac{360}{c} w\right]-\frac{50}{12} \Gamma_{L}\left[h_{0}, b_{0}\right]+\frac{1}{\pi c} \frac{272}{10} \int d^{2} z w b_{0}+\ldots
\end{aligned}
$$


Once more using the saddle-point equations, we can rewrite this as

$$
\begin{aligned}
& W_{\text {conn }}^{W_{3}}[t, w]= \\
& \quad \frac{c}{12}\left(1-\frac{122}{c}+\ldots\right) W_{L}\left[\frac{12}{c}\left(1+\frac{50}{c}+\ldots\right) t, \frac{360}{c}\left(1+\frac{386}{5 c}+\ldots\right) w\right] .
\end{aligned}
$$

We thus find that the computed result for $W_{\text {conn }}^{W_{3}}[t, w]$ can be summarized by the simple formula (12.2). We now propose that the exact, all-order result for this functional can be gotten by simply completing the $1 / c$ expansions indicated by the dots in (12.2). This leads to the formula

$$
W_{\text {conn }}^{W_{3}}[t, w]=2 k W_{L}\left[Z^{(t)} t, Z^{(w)} w\right]
$$

where $k, Z^{(t)}$ and $Z^{(w)}$ are functions of $c$ that allow the $1 / c$ expansions

$$
\begin{aligned}
k & =\frac{c}{24}\left(1-\frac{122}{c}+\ldots\right) \\
Z^{(t)} & =\frac{12}{c}\left(1+\frac{50}{c}+\ldots\right) \\
Z^{(w)} & =\frac{360}{c}\left(1+\frac{386}{5 c}+\ldots\right) .
\end{aligned}
$$

We remark that, the result for $k$ is consistent (in the classical limit $c \rightarrow-\infty$ ) with the formula

$$
k=-\frac{1}{48}(50-c+\sqrt{(c-2)(c-98)})-3,
$$

which is the conjectured outcome of a KPZ type analysis of constraints in a more covariant formulation of $W_{3}$ gravity $[38,20]$. For the two $Z$ factors, the following allorder resuits have been proposed ([39])

$$
Z^{(t)}=\frac{1}{2(k+3)}, \quad Z^{(w)}=\frac{\sqrt{30}}{\sqrt{\beta}(k+3)^{3 / 2}}
$$

with $\beta$ given in (2.3). They correctly reproduce the singularity structure that one expects, and are in agreement with the expansions (12.4). 
For pure gravity formulas similar to (12.3), (12.5) and (12.6) were proposed in [31] on the basis of the results in [30]. It would be most interesting to try and prove the proposal (12.6) on the basis of a similar analysis of $W_{3}$.

In general quantum field theories, an extrapolation from (partial) 1-loop results to an all-order result such as (12.3) for the effective action is not at all possible. Clearly, the quantum theory of chiral $W_{3}$ gravity enjoys miraculous features that are a consequence of its quantum integrability. It is especially appealing that the rather complicated underlying symmetry structuse, being nonlinear and infinite dimensional and falling outside the traditional class of finitely generated Lie algebras, in the end leads to transparent results such as (12.3) for the effective action of the fully quantized theory.

\section{Divergence equations and Tini Veltman.}

We have extensively used (anomalous) conservation equations for the currents $u$ and $v$ to obtain the quantum theory of $W_{3}$ gravity. It is perhaps appropriate to recall how Tini founded his research of quantum Yang-Mills theory on such current divergences.

Tini, in 1966 at Brookhaven, wrote an important paper, of which we reproduce the first page, in which he replaced current commutation rules by divergence equations. This eliminated Schwinger term difficulties. He was able to reproduce the known collection of results from current algebra, in particular the Adler-Weisberger relation. A few months later John Bell showed that these divergence equations can be derived from a theory with a gauge invariance. That was later a reason for Tini to start working on Yang-Mills theories.

In 1966, in London, Tini demonstrated that as a consequence of the divergence equations the decay of the neutral pion in photons was forbidden. John Bell, in the audience, started to work on that. First he stimulated Sutherland to prove this result using current algebra. Since then this forbiddenness is known as the Veltman-Sutherland theorem. Continued work from Bell with Jackiw led to the discovery of the anomaly, independently discovered by Adler as well.

Tini's first paper on massive Yang-Mills theories was published in 1968. It was thought at the time that the theory was not renormalizable, for example Salam and Komar had demonstrated in 1960 that the one-loop three point function contained non-renormalizable infinities. These divergences were caused by the $k k / M^{2}$ term in 
the vector boson propagator; no one knew how to get rid of these $k k / M^{2}$ terms. Tini invented a technique (a free field technique, or as called frivolously by Tini, the 'BellTreiman transformation'), to get rid of these $k k / M^{2}$ terms, and thus to implement the cancellation of many divergences. This technique consisted in taking a free scalar field, and performing a canonical transformation (which does not change the S-matrix, an obvious but important fact) such that it became interacting in such a way that many divergences in loop diagrams canceled. Since the Yang-Mills bosons were still massive, this was not a gauge transformation, but it looked very much like going from the unitary to the renormalizable gauge. The crucial observation by Tini was that one could go to different (renormalizable) formulations ("gauges", although they were really canonical transformations). All this was still at the one-loop level. The breaking of the gauge invariance by the $W$ mass terms led to non-renormalizable divergences at two loops.

Thus renormalizability (by power counting) was proven up to one loop diagrams. This paper revived interest in field theory of vector bosons and stimulated a number of authors (GIM for example), in particular Boulware, Fradkin and Tyutin in 1969. They reformulatec the techniques in the context of path integrals, rederiving the same results. Like Tini, they saw one-loop renormalizability at the one-loop level and nonrenormalizable divergences at the two-loop level. (Actually, Fradkin and Tyutin thought the theory was completely renormalizable).

A subsequent very important contribution of Fradkin and Tyutin was the application of the free field technique (in path integral setting) to the massless theory. This enabled them to formulate Feynman rules in various gauges. The earlier famous paper of Faddeev and Popov (1967) derives the rules in the Landau gauge. (Tini received this paper as an editor to Physics Letters B. He did not understand the paper, and hesitated for a while, but then decided to accept it anyhow. Fortunately. Just imagine...) Through the work of De Witt (1964) and Mandelstam (1968) the rules were also known in the Feynman gauge. It is this paper of Fradkin and Tyutin that led 't Hooft later to his general gauge formulation for the massless case; he derived the recipe with gauge-fixing and ghost lagrangian as we know today.

The issue of massless versus massive Yang-Mills theory was initially (1968) not well understood. Irituitively one thought that the massless theory would obtain from the massive in the limit of zero mass. This suggested that the $k k / M^{2}$ terms were 
probably harmless. The situation was clarified in 1969-1970 by Faddeev and Slavnov, and Tini with Henk Van Dam; the limit of zero mass of the massive theory turns out to be discretely different from the massless theory. This made it clear why the massless theory was renormalizable while the massive was not (at least at two loops and beyond).

So by the beginning of the seventies many of the ingredients for a successful future quantum field theory of the weak and strong interactions had been found: Yang-Mills theory, currents, diagrammatic techniques, one-loop renormalizability. In addition there was the paper of Gell-Mann and Levy on the sigma model, PCAC and spontaneous symmetry breaking. Many students in Utrecht had to study this article. Yet it was $\mathrm{n}$.t. clear how to combine all these concepts, and most physicists did not appreciate the fundamental importance of the results obtained.

We now reach the beginning of the seventies, when Tini and Gerard did their important discoveries on regularization, renormalizability and unitarity of the Yang-Mills theory. Their work, together with the classical theory of Yang and Mills, has become the basis of particle physics. The radiative corrections which follow from their work, are observed in large accelerators around the world.

\section{References}

[1] K. Schoutens, A. Sevrin and P. van Nieuwenhuizen, Phys.Lett. 243B (1990) 245

[2] K. Schoutens, A. Sevrin and P. van Nieuwenhuizen, Nucl.Phys. B349 (1991) 791

[3] K. Schoutens, A. Sevrin and P. van Nieuwenhuizen, in Proceedings of the Trieste conference on 'Topological methods in field theory', Int.Jour.Mod.Phys. A6 (1991) 2891

[4] K. Schoutens, A. Sevrin and P. van Nieuwenhuizen, Nucl.Phys. B364 (1991) 584

[5] H. Ooguri, K. S'choutens, A. Sevrin and P. van Nieuwenhuizen, The Induced Action of $W_{3}$ Gravity, preprint ITP-SB-91-16, RIMS-764, Comm.Math.Phys. to be published

[6] K. Schoutens, A. Sevrin and P. van Nieuwenhuizen, On the Effective Action of Chiral $W_{3}$ Gravity, preprint ITP-SB-91-21, Nucl.Phys. B to be published 
[7] A. van de Ven, Two-loop Quantum Gravity, preprint ITP-SB-91-52

[8] M.H. Goroff and A. Sagnotti, Phys.Lett. 160B (1985) 81

[9] K. Schoutens, A. Sevrin and P. van Nieuwenhuizen, Phys.Lett. 255B (1991) 549

[10] A.B. Zamolodchikov, Theor.Math.Phys. 63 (1985) 1205

[11] C.M. Hull, Phys.Lett. 240B (1990) 110; Nucl.Phys. B353 (1991) 707

[12] A. Belavin, Adv.Stud. in Pure Math. 19 (1989) 117

[13] A. Bilal and J.-L. Gervais, Phys.Lett. 206B (1988) 412; Nucl.Phys. B314 (1989) 646; Nucl.Phys. B318 (1989) 579; in 'Infinite dimensional Lie algebras and Lie groups', V.G. Kac editor (World Scientific, 1988), p. 483

[14] K. Li, Nucl.Phys. B346 (1990) 329; Phys.Lett. 251B (1990) 54

[15] E. Bergshoeff, C.N. Pope, L.J. Romans, E. Sezgin, X. Shen and K.S. Stelle, Phys.Lett. 243B (1990) 350;

[16] K. Schoutens, A. Sevrin and P. van Nieuwenhuizen, Phys.Lett. 251B (1990) 355

[17] E. Bergshoeff, P.S. Howe, C.N. Pope, E. Sezgin, X. Shen and K.S. Stelle, Nucl.Phys. B363 (1991) 163.

[18] C.N. Pope, L.J. Romans and X. Shen, Phys.Lett. 236B (1990) 173; Nucl.Phys. B339 (1990) 191.

[19] F. Bastianelli, An Action for Super-W $W_{3}$ Gravity, preprint ITP-SB-90-91

[20] Y. Matsuo, Phys.Lett. 227 B (1989) 209; in proceedings of the meeting 'Geometry and Physics', Lake Tahoe, July 1989

[21] C.M. Hull, $W$ Gravity Anomalies 1: Induced Quantum $W$ Gravity, preprint QMW/PH/91/2; Phys.Lett. 265B (1991) 347.

[22] K. Schoutens, A. Sevrin and P. van Nieuwenhuizen, in Proceedings of the Jan. 1991 Miami Workshop on 'Quantum Field Theory, Statistical Mechanics, Quantum Groups and Topology' (Plenum, 1991) 
[23] A.T. Ceresole, M. Frau, J. McCarthy and A. Lerda, Phys.Lett. 265B (1991) 72

[24] K. Li and C. Pope, in Proceedings of the Trieste Summer High-Energy Workshop, July 1990

[25] C.M. Hull, $W$ Gravity Anomalies with Ghost Loops and Background Charges, preprint QMW/PH/91/14; C.M. Hull and L. Palacios, $W$ algebra Realisations and $W$ Gravity Anomalies, preprint QMW/PH/91/15.

[26] J. Thierry-Mieg, Phys.Lett. 197B (1987) 368; K. Schoutens, A. Sevrin and P. van Nieuwenhuizen, Comm.Math.Phys. 124 (1988) 87;

[27] L.J. Romans, Nucl.Phys. B352 (1991) 829

[28] C.N. Pope, L.J. Romans and K.S. Stelle, Phys.Lett. 268B (1991) 167

[29] A.M. Polyakov, Mod.Phys.Lett. A2 (1987) 893; in Proceedings of the Les Houches 1988 meeting on 'Fields, Strings and Critical Phenomena' (Elsevięr, 1989)

[30] V.G. Knizhnik, A.M. Polyakov and A.B. Zamolodchikov, Mod.Phys.Lett. A3 (1988) 819

[31] Al.B. Zamolodchikov, Liouville Action in the Cone Gauge, preprint ITEP 84-89 (1989)

[32] A. Bilal and J.-L. Gervais, Nucl.Phys. B326 (1989) 222

[33] S.R. Das, A. Dhar and K.S. Rama, Physical properties of $W$ gravities and $W$ strings, preprint TIFR/TH/91-11; Physical states and scaling properties of $W$ gravities and $W$ strings, preprint TIFR/TH/91-20

[34] C.N. Pope, L.J. Romans and K.S. Stelle, On $W_{3}$ strings, preprint CERNTH.6188/91; C.N. Fope, L.J. Romans, E. Sezgin and K.S. Stelle, The $W_{3}$ string spectrum, preprint CPT TAMU-68/91

[35] A.A. Belavin, A.M. Polyakov and A.B. Zamolodchikov, Nucl.Phys. B241 (1984) 333 
[36] F.A. Bais, P. Bouwknegt, K. Schoutens and M. Surridge, Nucl.Phys. B304 (1988) 348

[37] J. Pawelczyk, Phys.Lett. 255B (1991) 330

[38] M. Bershadsky and H. Ooguri, Comm.Math.Phys. 126 (1989) 49

[39] J. de Boer, private communication 

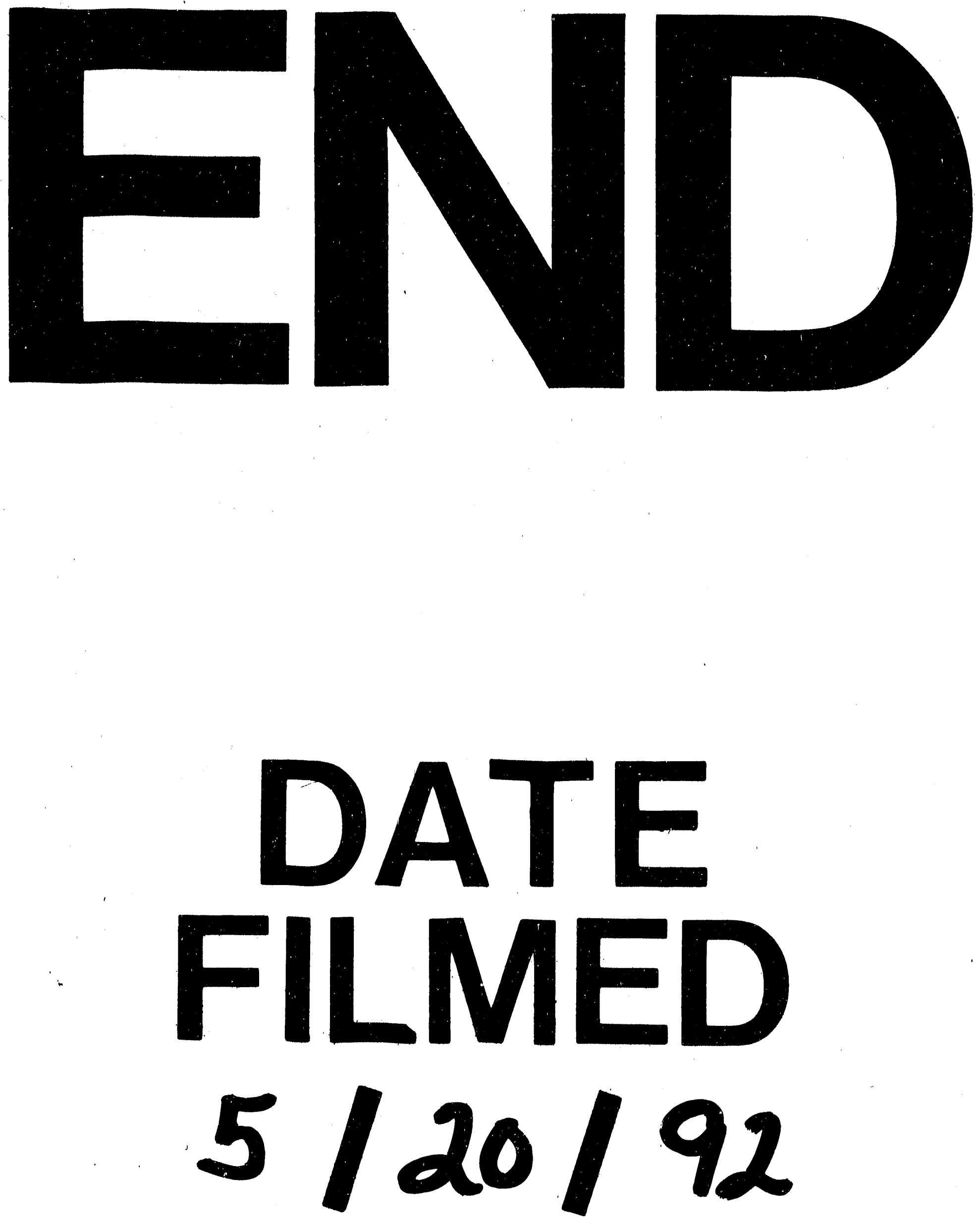

1 
